\title{
Neurodevelopmental disorders in youth justice: a systematic review of screening, assessment and interventions
}

\author{
Lorelle Holland $^{1,2,3}$ (D) Natasha Reid $^{4}$ (D) $\cdot$ Andrew Smirnov ${ }^{5,6}$ (D)
}

Accepted: 20 May 2021 / Published online: 21 July 2021

(C) The Author(s) 2021

\section{Abstract}

Objectives This study aims to systematically review screening and assessment approaches and therapeutic interventions for young people with neurodevelopmental disorders (NDDs) in the youth justice system (YJS).

Method Five databases were searched (PubMed, Embase, CINAHL, Web of Science and PsycINFO) for studies evaluating effectiveness of screening, assessment or interventions, targeting people aged 10-18 years in youth justice with a NDD.

Results Eighteen peer-reviewed articles ( 9 screening/assessment; 9 intervention studies) were eligible. More comprehensive assessment appeared to be superior to brief screening for identifying NDDs. Interventions addressed social and emotional wellbeing, educational, employment, language and communication and behavioural outcomes, community engagement, quality of life factors and recidivism. Limited consideration was given to cultural diversity in evaluations of screening, assessment or interventions.

Conclusions The limited amount of evidence retrieved suggests further research is urgently needed in the provision of culturally competent and holistic screening, assessment and treatment for young people with NDDs in the YJS.

Keywords Assessment · Interventions · Neurodevelopmental disorder · Screening · Systematic Review · Youth justice system

Emerging evidence describes the disproportionate representation of children with neurodevelopmental disorders (NDDs) in the youth justice system (YJS;

Lorelle Holland

lorelle.holland@uq.edu.au

Extended author information available on the last page of the article 
Chitsabesan et al., 2014; Hughes et al., 2017). Advocates and researchers have pointed to the human rights and practical imperatives of improving care for these children and question the appropriateness of their detention (Hughes et al., 2020; McSherry et al., 2017; Tedeschi and Junewicz, 2018). Disproportionate global incarceration rates may be partly due to a failure to provide timely, comprehensive and multidisciplinary screening, assessment and intervention for identifying and supporting children with NDDs who are exposed to the YJS (Lennox, 2014). A range of interventions may be required to improve quality of life and reduce or circumvent detention practices and recidivism during critical periods of child development, including vocational and educational support, health services, diversion and welfare assistance (Gilman et al., 2015; Arthur, 2016; Baldry et al., 2018; McCausland and Baldry, 2017). For example, multidisciplinary approaches may facilitate more rigorous screening and assessment, potentially improving the identification of young people with NDDs in the YJS and increasing opportunities to divert these young people away from the YJS or to deliver therapeutic support and interventions (Lennox, 2014; Tedeschi and Junewicz, 2018). However, there is a lack of understanding of the effectiveness of available interventions for identifying young people with NDDs in the YJS and promoting better outcomes (Baker, 2017; Lerner et al., 2012). Little is known about the appropriate design of interventions for specific NDDs or the ways in which interventions may address the underlying societal factors for these young people.

\section{Neurodevelopmental disorders}

Neurodevelopmental disorder is an umbrella term for conditions in which the determining feature is disruption to brain development during critical embryonic and childhood developmental periods that occur prior to puberty (Thapar et al., 2017). Aetiologic factors of NDDs include genetic, prenatal exposure to teratogenic drugs or substances, prenatal or birth trauma and childhood injury or illness (Hughes et al., 2012). The Diagnostic and Statistical Manual of Mental Disorders Fifth Edition (DSM-5) categorises NDDs together as conditions that limit a child's ability to meet expected developmental milestones and exhibit particular clinical symptoms that may present as both excesses and deficits in functioning (American Psychiatric Association, 2013). NDDs include conditions such as: intellectual disability (ID), attention deficit hyperactivity disorder (ADHD), autism spectrum disorder (ASD), traumatic brain injury (TBI), fetal alcohol spectrum disorder (FASD), developmental language disorder (DLD) and specific learning disorder (SLD).

Developmental deficits related to NDDs include the following: learning difficulties, reduced control of executive functioning (e.g. effective decision making, attention, purposeful and well-focused behaviour and impulse control) and global impairments of intelligence, memory, adaptive functioning (e.g. everyday life skills) or social skills that ultimately impact upon aspects of social, occupational, academic and personal performance throughout a child's life (American Psychiatric Association, 2013). Difficulties experienced by 
children with a particular NDD are exacerbated by the frequent comorbidity of NDDs. For example, children with ADHD commonly experience specific learning disorders (American Psychiatric Association, 2013). Specific NDDs and related impairments contribute to life-long negative impacts that may increase criminogenic risk for young people and consequent exposure with the youth justice system (YJS; Mohr-Jensen et al., 2019; Hughes et al., 2012, 2017).

ADHD, is a condition that involves symptoms of pervasive inattention and/or hyperactivity and impulsivity that are excessive for a child's developmental age (Young et al., 2018), which occurs in the general youth population at 5\% compared with $18.5 \%$ for females and $11.7 \%$ for males in youth detention populations (Hughes et al., 2012). ASD comprises symptomatology that is demonstrated by deficits of social communication that coexist with excessive repetitive behaviours and strong desirability for routine and familiarity (American Psychiatric Association, 2013). ASD has a 0.6 to $1.2 \%$ prevalence in the general youth population compared with $15 \%$ prevalence in the youth detention population (Hughes et al., 2012). Additionally, TBI also has a high prevalence of $65-75 \%$ in youth detention compared with $5-24 \%$ in the general youth population (Hughes et al., 2012).

Further examples of NDDs that are disproportionately represented in the YJS are DLD, FASD and ID. DLD is classified according to the area of impairment, which includes severe delays in articulation of speech, expressive language, receptive language (comprehension) and dysfluency, negatively impacting academic achievement, social relationships and quality of life (Baird, 2008; Snow and Powell, 2008, 2011). DLD has a 1-7\% prevalence rate in the general youth population compared with $60-90 \%$ in the youth detention population (Hughes et al., 2012). FASD is the result of prenatal alcohol exposure and includes a wide range of neurodevelopmental impairments (e.g. cognitive, behavioural and learning) and physical impairments (e.g. growth, distinct facial dysmorphology; Bower and Elliott, 2016; Baker, 2017; Burd et al., 2010; Passmore et al., 2016). A recent Australian study reported 36\% prevalence of FASD for children in the youth detention population (Bower et al., 2018). Three criteria need to be met to determine a diagnosis of ID, a significant impairment in intellectual functioning, adaptive functioning and the onset occurring before the age of 18 years (McKenzie et al., 2012). ID has a prevalence of 2 to $3 \%$ in the general youth population and 7 to $25 \%$ in the youth detention population (Hughes et al., 2012). With overlapping co-occurring NDDs and complex symptomatology experienced by these children, it is also important to consider the need for standardised interdisciplinary screening, assessment and intervention strategies.

\section{Theoretical frameworks to guide practice}

Given the vulnerability of young people with NDDs to be exposed to - and ensnared in-the criminal justice system, and the likely harmful consequences of this exposure (Gilman et al., 2015; Human Rights Watch, 2018; Hughes et al., 2020; Kippin et al., 2018), there is an urgent need for systemic changes 
to protect marginalised youth. Merging contemporary critical disability and critical criminological theories provides a holistic hybrid framework for understanding the over-representation of young people with NDDs in the YJS and the need to formulate appropriate early childhood prevention strategies, intervention programs and policy responses (Akers et al., 2017). 'Disabling critical criminology' is a hybrid theory that merges critical criminology, emphasising the relationship between the individual and structural and institutional contexts of crime, and critical disability theory, which seek to move beyond the medicalisation of disability, by examining societal and systemic factors that lead to stigmatisation and social exclusion of people with disability (Dowse et al., 2009, p. 29). Disabling critical criminology theory drives a global political agenda that recognises the need to have higher thresholds of inclusion and equality and to eliminate human rights violations, such as punishment and incarceration of disabled youth for the purpose of social control (Dowse et al., 2009; DeKeseredy and Perry, 2006; Reid-Cunningham and Fleming, 2009). Baldry et al. (2018, p. 639) builds upon this theoretical framework by describing the need to holistically address the significant intertwined impacts 'between individuals, institutions and systems that lead to disadvantaged and marginalised young people with disability (and/or complex needs) being funnelled into, around and often back into, youth justice systems before eventually being discharged into adult prisons'. Disabling critical criminology provides a framework for improving outcomes for disabled youth, through interdisciplinary collaboration that is founded upon a socially informed understanding of the holistic needs of children with NDDs, inclusive of their biological, socialemotional and cultural needs. In contrast to these perspectives calling for YJS reform, other theorists point to the inherent violence of imprisonment and have argued, more radically, for the abolition of youth detention (Brown and Schept, 2017; Goldson, 2005). Given the recognised human rights imperative of eliminating the social exclusion and imprisonment of young people with NDDs, the abolition of youth detention could be considered an ideal state, to the extent that appropriate support, interventions and alternative pathways for young people are provided. Consequently, our understanding of screening, assessment and intervention in this review is guided by the holistic intersection of critical disability theory, critical criminology and abolitionist perspectives.

\section{Social determinants, youth disadvantage and trauma}

Disabling critical criminology highlights the institutional, structural and societal aspects of the involvement of young people in the YJS, to inform appropriate reform and interventions. Young people with a NDD exposed to the YJS are subject to a social gradient whereby their negative health outcomes may be exacerbated by their experience of relative social disadvantage (Abramovitz and Mingus, 2016; Allen et al., 2014; Baldry et al., 2018). Youth with NDDs are frequently impacted by these determinants and risk factors that include prenatal fetal exposure to harmful licit and illicit substances, dysfunctional family dynamics, limited education, childhood stress associated with traumatic events, 
psychological, physical and sexual abuse, socioeconomic disadvantage, homelessness, substance abuse and mental health issues (Abramovitz and Mingus, 2016; Ford and Blaustein, 2013; Maughan, 2017; Reid, 2018). In addition, structural racism may exacerbate unequal health and social outcomes for young people with NDDs (Bailey et al., 2017).

Racially discriminatory practices arising from prolific colonisation of Indigenous peoples and lands perpetuate disparities that contribute to health and social disadvantage experienced by Indigenous youth (Baker, 2017; Bond and Singh, 2020; Abramovitz and Mingus, 2016). Inequitable positioning of Indigenous children around the globe is reflected in the over-representation of Indigenous youth incarcerated (Lennox, 2014; McCausland and Baldry, 2017). In 2018, in Australia, a daily average of almost $59 \%$ or 3 in 5 Aboriginal and Torres Strait Islander youth aged between 10 and 17 years old (who represent $5 \%$ of the Australian population) were detained in care; this accounts for an incarceration rate that is 26 times greater than for Australian youth of other descent (Australian Institute of Health and Welfare, 2018; Yi and Foley, 2014). These incarceration rates of Indigenous youth are paralleled in Canada, New Zealand and in the USA (Pfeifer et al., 2018). Consequently, it is important to consider whether screening, assessment methods and interventions are socially and culturally appropriate. Trauma-informed approaches may also be an important part of interdisciplinary practice to enhance holistic, youth-centred care for young people with NDDs who are exposed to the YJS.

\section{Self-determination and youth-centred approaches}

There are some examples globally of innovative programs, which acknowledge institutional, societal and structural issues, and are responsive to the needs of young people with NDD, including their cultural needs. Given the abuses of youth justice systems, a contemporary approach requires reformed legal frameworks inclusive of collaborative interdisciplinary contributions from within 'legal, psychiatric, and developmental fields' (Young et al., 2017, p. 26). For instance, the New Zealand Youth Courts is a solution-focused justice approach that facilitates inter-agency collaboration to improve outcomes for young people with NDDs (Fitzgerald, 2019). Legislative changes occurred in 1989 with the establishment of The Oranga Tamariki Act (OTA) and the introduction of the Youth Court (the Court; Fitzgerald, 2019). These courts adhere to the same laws as mainstream courts, but embrace Maori customs, protocols and language to acknowledge the loss of cultural identity for youth offenders and enhance cultural safety and mutual respect by all parties contributing to the youth-centred judicial process (Fitzgerald, 2019). The pioneering efforts offered within the New Zealand framework acts to minimise further trauma and social marginalisation of youth with NDDs, including those who are from minority and culturally diverse backgrounds exposed to the YJS (Hughes et al., 2020; Human Rights Watch, 2018; United Nations, 1989, 2006, 2007; Maughan, 2017).

Other Indigenous peoples have also actively sought to address, through selfdetermination and pursuit of culturally responsive strategies, the over- 
representation and mistreatment of their youth in the criminal justice system. The resilience and strength of Native American youth, their families and their communities is demonstrated by their survival, resistance and a thriving population growth that opposes centuries of enforced assimilation policies (Seelau, 2012). Despite recognising statistical data that outlines the negative plight of Native American youth, including unequal health and educational outcomes and disproportionate incarceration rates in comparison to their non-Indigenous peers, communities have engaged in self-determining strategies to enact long-lasting preservation of culture to nurture their youth, holistic wellbeing and intergenerational healing (Seelau, 2012). Seelau (2012, p. 65) suggests the answer to counter youth detention lies within 'meaningful self-determination and the ability of Native nations to exercise true sovereignty over their own children and the issues that affect them'. It is apparent that Indigenous youth deserve nurturing care by remaining with their own family and in their own communities, with Indigenous-led intersectoral community-level support, to counter youth detention practices (United Nations, 1989, 2006, 2007). However, without consistent international best practice to identify and support young people with NDDs who are exposed to the YJS a range of continued negative consequences are inevitable.

\section{Consequences of failing to identify neurodevelopmental impairment}

The detrimental impacts of not identifying youth with NDDs are that evidencedbased therapeutic care and interventions, appropriate rehabilitation, welfare and diversion for these vulnerable youth may be inadvertently neglected (Williams, 2012; Young et al., 2017; Tedeschi and Junewicz, 2018). Additionally, their neurodevelopmental impairments will likely remain unknown during engagement with police, lawyers, judges and prison staff (Williams, 2012; Young et al., 2017; Tedeschi and Junewicz, 2018). Young people with NDDs may not possess the required interpersonal and cognitive skills to facilitate self-defence and understand presumed innocence, which reduces their capacity to preserve their legal rights of justice during interrogation by police and during judicial processes (Hughes et al., 2020; McCausland and Baldry, 2017; Reid et al., 2020). This may lead to extended periods of incarceration rather than appropriate alternative communitybased holistic supports, with possible implications for wellbeing and recidivism rates (Baldry et al., 2018). Neglecting complex needs creates an atmosphere in which control, punitive management and punishment of youth with NDDs may be accepted and negates the United Nations principles of human rights for children with disabilities (Arthur, 2016; McCausland and Baldry, 2017; United Nations, 1989, 2006, 2007).

\section{The aim of the review}

To the authors' knowledge, there are no previous reviews investigating the effectiveness of screening, assessment and interventions for youth with NDDs in the YJS. Consequently, the current systematic review is informed by the 
necessity to identify appropriate and effective approaches to reduce detention and recidivism for young people with NDDs. The aim of the study is to systematically review and synthesise the available evidence regarding effective screening, assessment and interventions for this population. Our synthesis of the evidence is guided by a theoretical framework of disabling critical criminology and an understanding of the distinct and overlapping characteristics of different NDDs. Thus, we assess the extent to which interventions address the complex health needs of young people with NDDs inclusive of challenging behavioural manifestations, neurocognitive, learning, speech and language impairments, diminished cultural empowerment, racial disparities and social determinants that increase the risk of criminality.

\section{Methods}

\section{Registration and search strategy}

The current review was registered with the international prospective register of systematic reviews in April 2020 (PROSPERO, CRD42020140527) and complies with the Preferred Reporting Items for Systematic Reviews and MetaAnalyses (PRISMA) statement (Moher et al., 2009). A systematic search was conducted to identify relevant articles across five bibliographic databases (PubMed, Embase, CINAHL, Web of Science and PsycINFO) from inception to July 2019, completed by the first author (L.H). We anticipated there would be limited available research on this topic, and consequently we did not apply any year limits, so that we could capture as many potential studies as possible. An updated search was undertaken before submission (23 Nov. 2020). Key phrases and synonyms pertaining to the target population (youth with NDDs), YJS, screening, assessment and interventions were used (Supplementary Data File 1 provides the search strategy applied for each database).

\section{Inclusion and exclusion criteria}

Articles were included if they met the following criteria: (a) young person aged 10-18 years involved with the YJS and who had a NDD, inclusive of ADHD, ASD, TBI, FASD, ID and SLD and synonyms (see Supplementary Data File 1 for the full list of NDD terms); (b) studies that evaluated screening and assessment tools or outcomes of interventions regardless of study design; (c) outcomes of interest included: improvements in the identification of NDDs, improvements in social and emotional wellbeing, reductions in challenging behaviours/behavioural problems, improvements in adaptive behaviour/ everyday life skills, increased employability, increased engagement in school and/or other educational activities, improved quality of life, youth satisfaction with interventions and reductions in youth detention and recidivism. Studies were excluded if they were (1) not in English, or (2) conference abstracts, editorials, grey literature, reviews, not original research, $\mathrm{PhD}$ theses or government reports. 


\section{Assessment of methodological quality}

A modified Downs and Black (1998) checklist was utilised to assess the quality of the studies. The Downs and Black checklist was developed to assess the methodological quality of randomised and non-randomised studies and contains 27 items (Downs and Black, 1998). Items 1-4 cover the reporting of hypothesis/aim/objective, participant characteristics, interventions (scored, Yes $=1$, No $=0$ ); item 5 measures the study's clear description of distributions of principal confounders in each group of subjects to be compared (scored, Yes $=2$, Partially $=1,0=$ No); items 6-10 measures findings, length of follow-up, adverse events of interventions, random variability of the main outcomes and probability (scored, Yes $=1$, No $=0$ ); items $11-13$ report external validity by assessing the representativeness of study participants to the entire recruitable population (scored, Yes $=1$, No $=0$, Unable to determine $=0$ ); internal validity is reported by assessing for bias through Items 14-20 (scored, Yes = 1 , No $=0$, Unable to determine $=0$ ); Internal validity is measured by Items 21 26 (scored, Yes $=1$, No $=0$, Unable to determine $=0$ ) and statistical power assessed by Item 27, scored according to statistical power calculations and sample sizes that have been calculated to detect a difference of $\mathrm{x} \%$ and $\mathrm{y} \%$ (ranked from A, B, C, D, E, F and scored 0-5, respectively).

Two authors (LH and AS) independently assessed all included studies. Differences in ratings were resolved by discussion. For screening and assessment studies, the tool was modified by removing all items that related only to interventions: Items 21 and 22 were amended to reflect that the participants were recruited from the same population setting and the same time period respectively, and item 27 was adapted by scoring the study according to whether a power calculation was reported, rather than scoring on the level of study power $(1=$ power calculation was conducted; $0=$ power calculation was not conducted). This reduced the total achievable score to 19 for the screening and assessment studies (see Supplementary Data File 2). For intervention studies, all 27 items were scored. Items 21 and 22 were amended to reflect that the participants were recruited from the same population setting and the same time period, respectively. Item 27 was adapted as described above. This reduced the total achievable score to 28 (see Supplementary Data File 2). Additionally, clinical trial registries and searches for study protocol documents were undertaken to check for any evidence of publication bias.

\section{Data extraction}

Data were extracted from each study by two authors (LH and NR). Data were extracted from screening and assessment studies according to the following predetermined parameters: study aim, study design and location, participant characteristics, index test, reference standard, test accuracy and key conclusions. Regarding the intervention studies, data were extracted according to the following pre-determined parameters: study aim, design and setting, study population and sample size, participant characteristics, period of follow-up, 
intervention, control and key outcomes and quantitative effect measures and key findings.

\section{Synthesis method}

A narrative approach was selected because the identified studies were clinically heterogeneous (Bland, 2015), with regard to the types of clinical research questions, interventions, study designs, sampling (including single case studies), and outcome measures that were used. Furthermore, the very small number of studies on specific types of NDDs limited our capacity to quantitatively summarise results for each condition; for three types of NDDs, only one intervention study was found, and there was a maximum of three intervention studies for any given condition. We also consider a narrative review approach to be well-suited to the task of critically exploring and enriching understanding of assessment and interventions for NDDs in youth justice settings, which is a nascent area of research (Greenhalgh et al., 2018). Available descriptive and inferential statistics from each study (including test accuracy in the case of screening and assessment studies) have been summarised within the tables, as have comparable outcome measures for specific NDDs, where available.

\section{Results}

\section{Search results}

The electronic database search retrieved 1970 potential citations after duplicates were removed. The titles and abstracts of retrieved articles were screened according to the inclusion and exclusion criteria. Forty-seven articles were considered for full text review and an additional four articles were found by searching reference lists. One additional article was identified following the updated search prior to submission. Eighteen articles met inclusion criteria for the current systematic review including: eight screening, one assessment and nine intervention studies. Specific reasons for exclusion are provided in the PRISMA Study Selection Flow Chart (Fig. 1).

An updated search (5/3/2021) was performed in Web of Science to provide an analysis of evidence retrieved to summarise the disciplinary contributions (338 articles). Seventeen out of the eighteen articles that met inclusion criteria could be retrieved in the Web of Science. Figure 2 demonstrates the broad range of disciplines that contributed to the current review.

\section{Quality of the methodological reporting}

Figure 3 and Supplementary Data File 2 provide a detailed summary of the quality assessment for the included screening and assessment studies. Scores were variable, ranging from 9 to 16 out of a total of 19 , with an average score of 11.62. Ford et al. (2008) obtained the lowest score of 9, out of a possible 19. The highest score of 16 , out of a possible 19 was obtained by Colins 


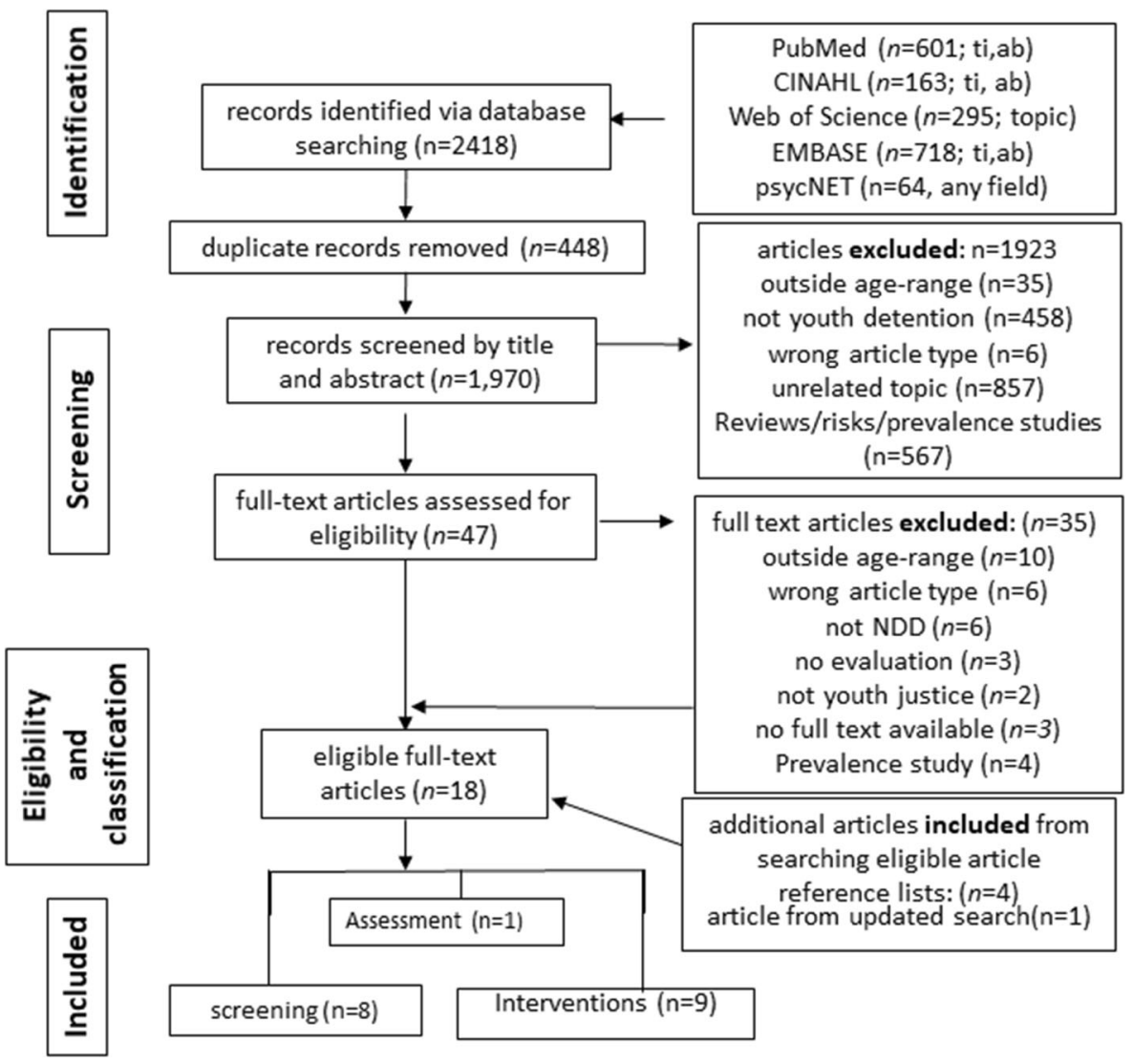

Fig. 1 PRISMA study selection flow chart (Moher et al., 2009)

(2016). Even considering the adjustments to the Downs and Black (1998) for appraisal of non-interventional studies, the scores for screening and assessment

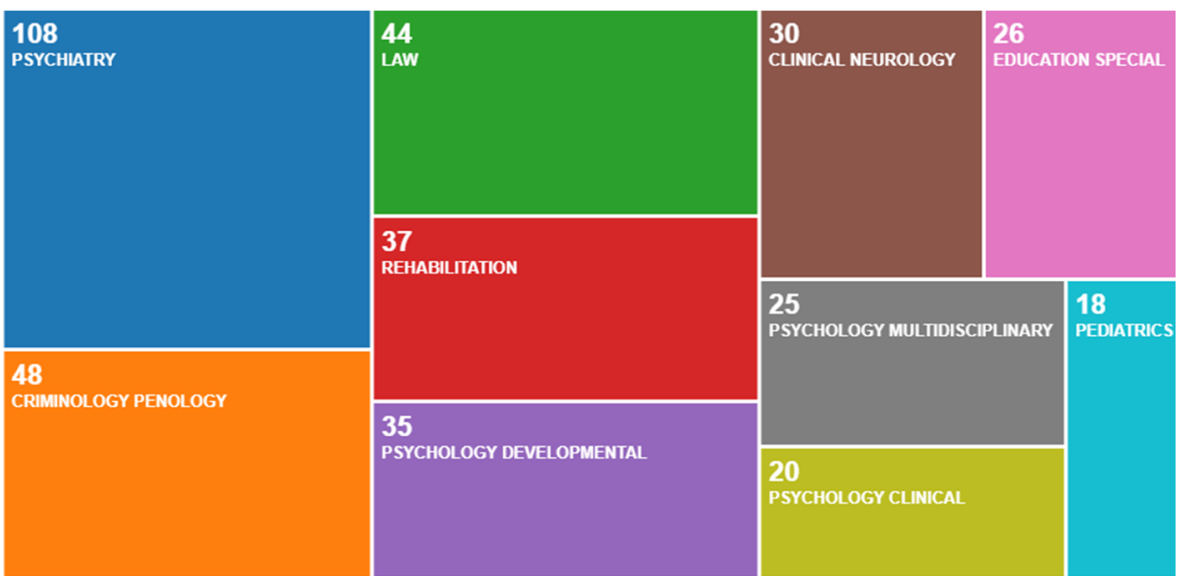

Fig. 2 Interdisciplinary contribution 


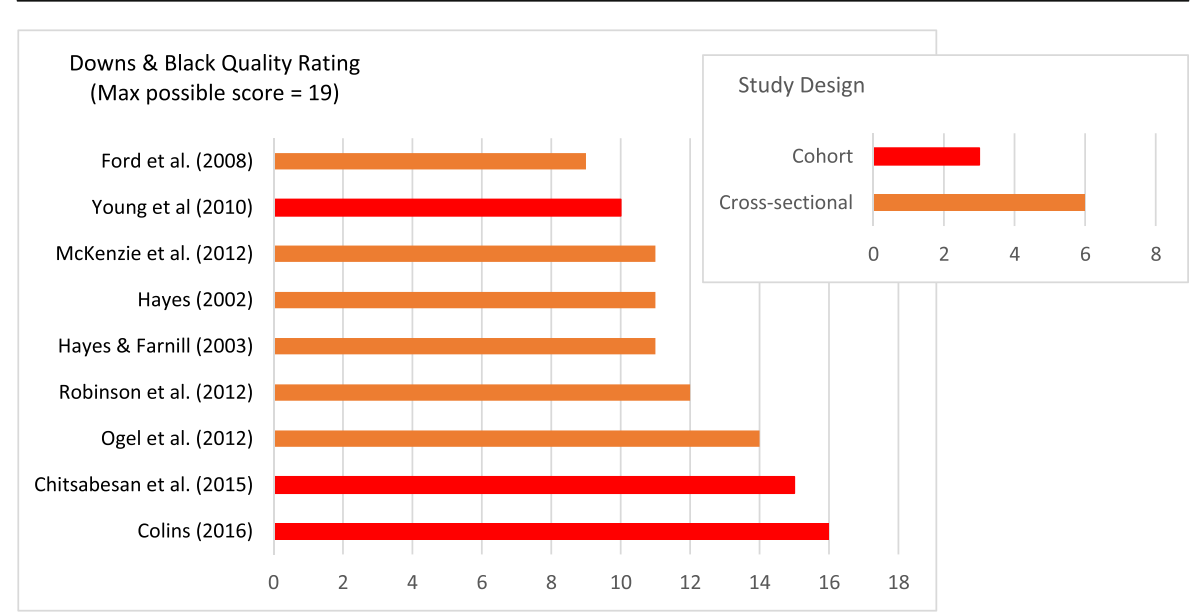

Fig. 3 Quality assessment scores for screening and assessment studies according to the Downs and Black assessment criteria (Downs and Black, 1998)

studies were relatively low. Scores were generally lower because of limited external validity, lack of reporting of confounders (item 5), use of convenience rather than random sampling, lack of reporting of recruitment procedures and non-response (items 11 and 12), lack of blinding of outcome assessors (item 15) and lack of control for confounders (item 25). Ögel et al. (2012) was the only study that considered confounding factors yet failed to provide a statistical analysis of these variables. Chitsabesan et al. (2014) was the only study that used random sampling methods of recruitment and appropriately reported this (items 11 and12). Only four studies blinded the measurement outcomes (item 15; Chitsabesan et al., 2014; McKenzie et al., 2012; Robinson et al., 2012; Young et al., 2010).

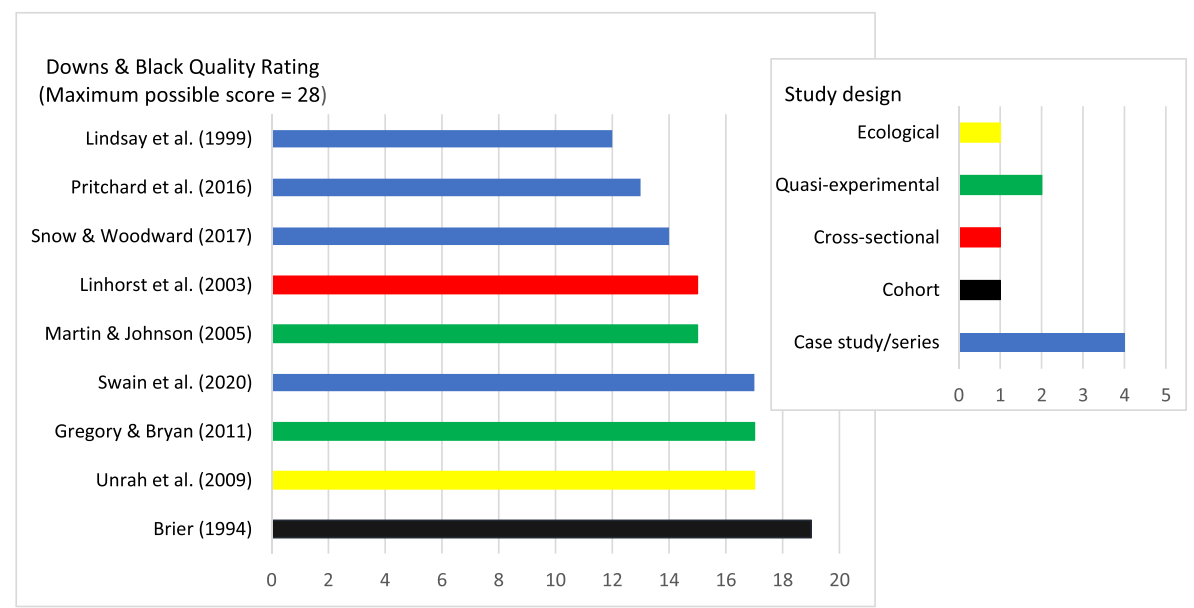

Fig. 4 Quality assessment scores for intervention studies according to the Downs and Black assessment criteria (Downs and Black, 1998) 


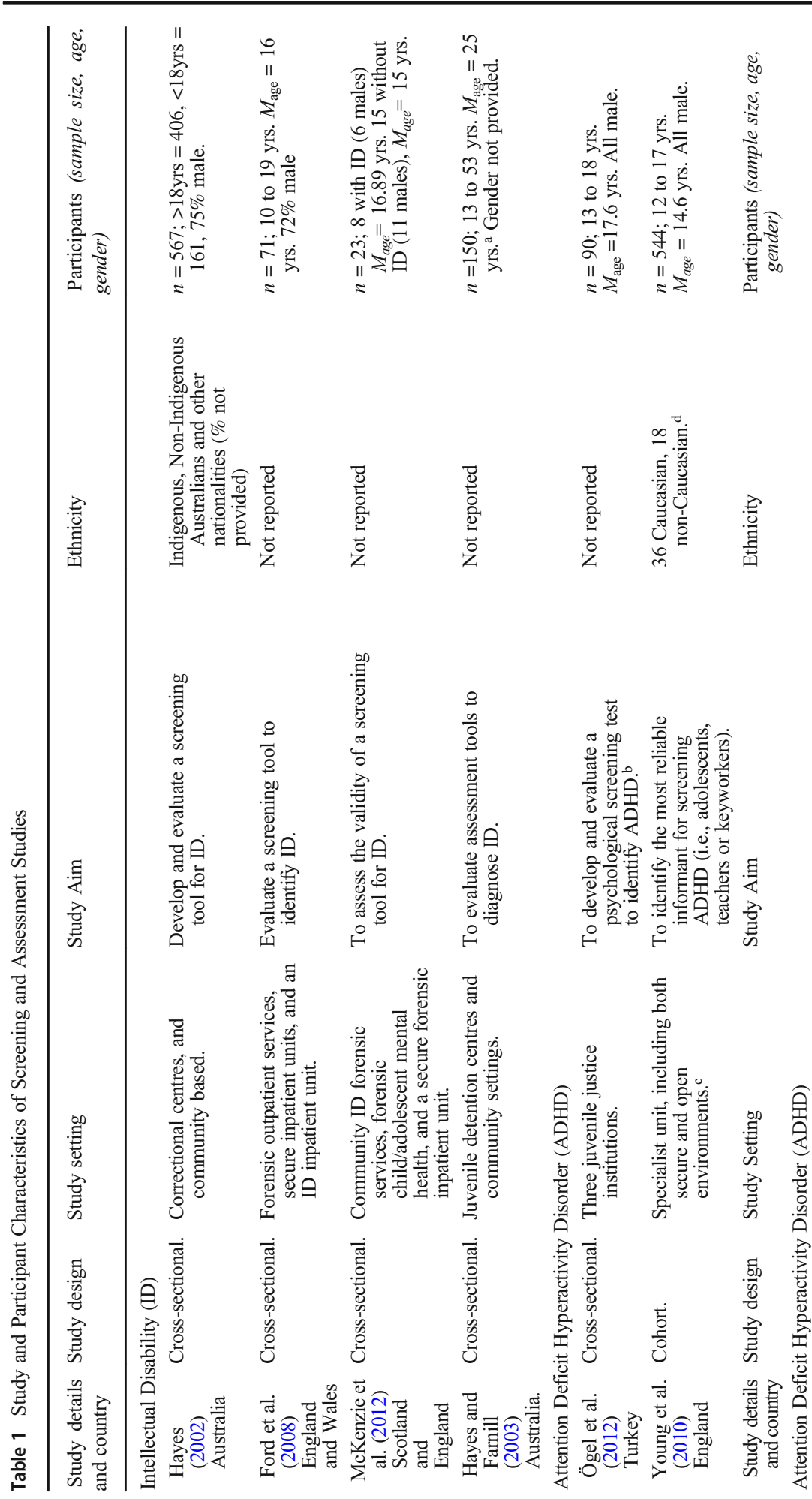




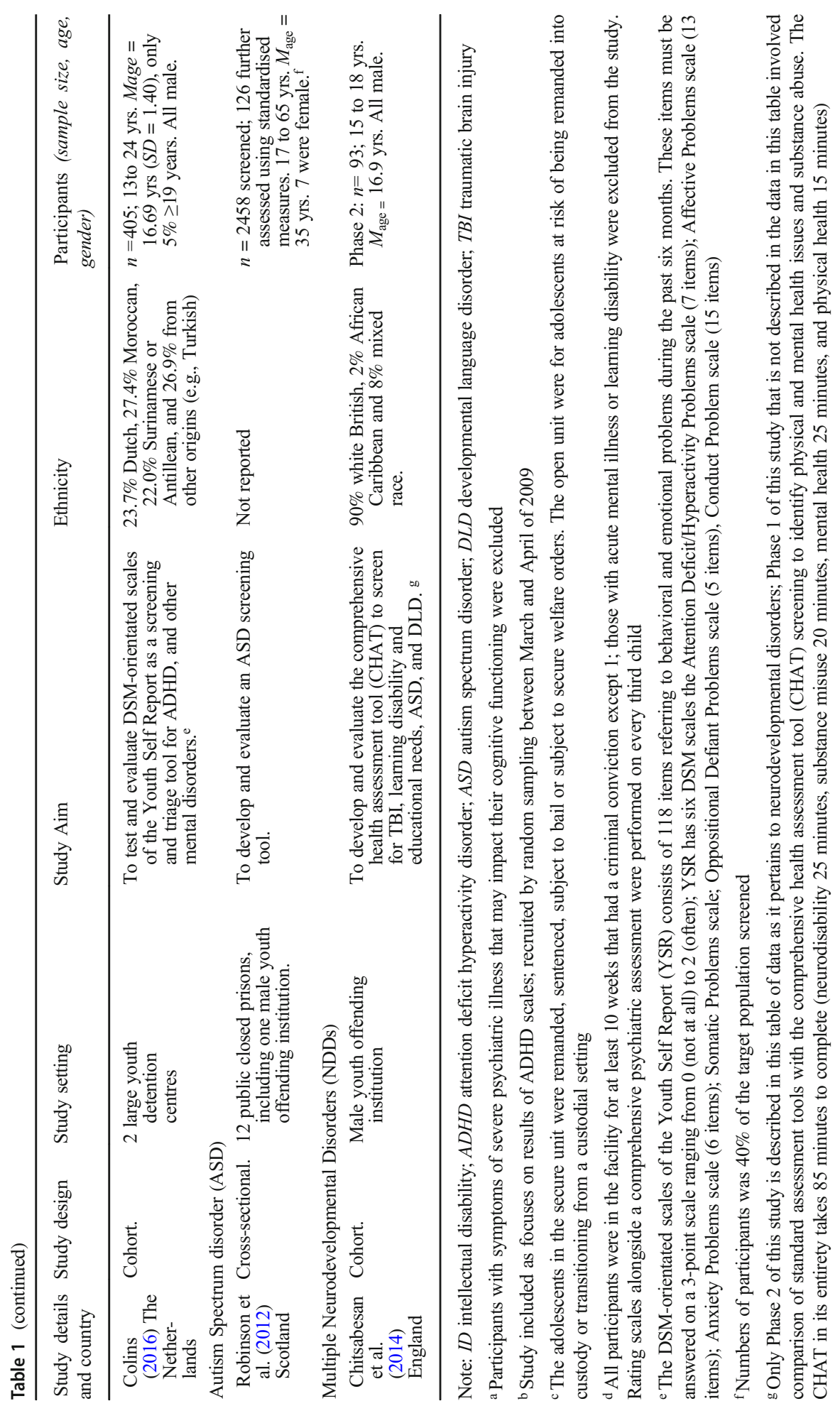


Figure 4 and Supplementary Data File 2 provide a detailed summary of the quality assessment for the included intervention studies. Scores ranged from 12 to 19 out of a total of 28 , with an average score of 15.44 . The case series by Lindsay et al. (1999) scored the lowest at 12. In comparison, the highest score obtained was 19 out of a possible 28 for the prospective longitudinal cohort study conducted by Brier (1994). Generally, lower scores were due to controlling for confounders (item 5), participant recruitment (convenience versus probabilistic sampling), explanation of proportions of participants recruited from particular settings (items 11 and 12) and statistical power of studies (Item 27). Additionally, no results were found following searches of trial registries, public protocol registration websites (i.e. Open Science) and peer-reviewed protocol documents.

\section{Participant ethnicity and socioeconomic status for screening and assessment studies}

Table 1 provides the participant details for screening and assessment studies. Details of ethnicity were not included in five out of nine studies (Ford et al., 2008; Hayes and Farnill, 2003; McKenzie et al., 2012; Ögel et al., 2012; Robinson et al., 2012). Two studies reported diverse ethnicities (Hayes, 2002; Young et al., 2010). Chitsabesan et al. (2014) described the participants as $90 \%$ white British, 2\% African Caribbean and 8\% mixed race. Colins (2016) reported 23.7\% Dutch, 27.4\% Moroccan, 22.0\% Surinamese or Antillean and $26.9 \%$ from other origins (e.g. Turkish). All studies provided details of participants' ages. Only one study comprehensively described social and demographic confounding variables, such as educational achievement, number of crimes committed, age when offences took place and the detention facility (Ögel et al., 2012). Few studies specifically focused on screening and assessment approaches for people from First Nations backgrounds.

\section{Summary of assessment and screening study outcomes}

Nine studies were identified that investigated screening and/or assessment approaches for identifying young people with NDDs in the YJS (Table 2). The majority of the studies demonstrate sensitivity and specificity in percentages with accompanying statistical analysis to ensure accuracy represented by the range of confidence intervals, ROC and AUC percentages, accuracy of data was reinforced when positive predictive values and negative predictive values was provided (Trevethan, 2017). The benefit of a screening tool relies on the premise that they are less time consuming and are able to be performed by non-professional staff and therefore, less costly (Trevethan, 2017). Additionally, if a screening test accurately detects a NDD then an appropriate referral can be made for further interdisciplinary standardised testing to detect need and required interventions. However, it is essential that screening and assessment tools are robust and rigorous and are statistically reliable and valid that appropriately guides clinical and policy decision making (Table 2 provides effect measures) (Trevethan, 2017; McKenzie et al., 2012). 


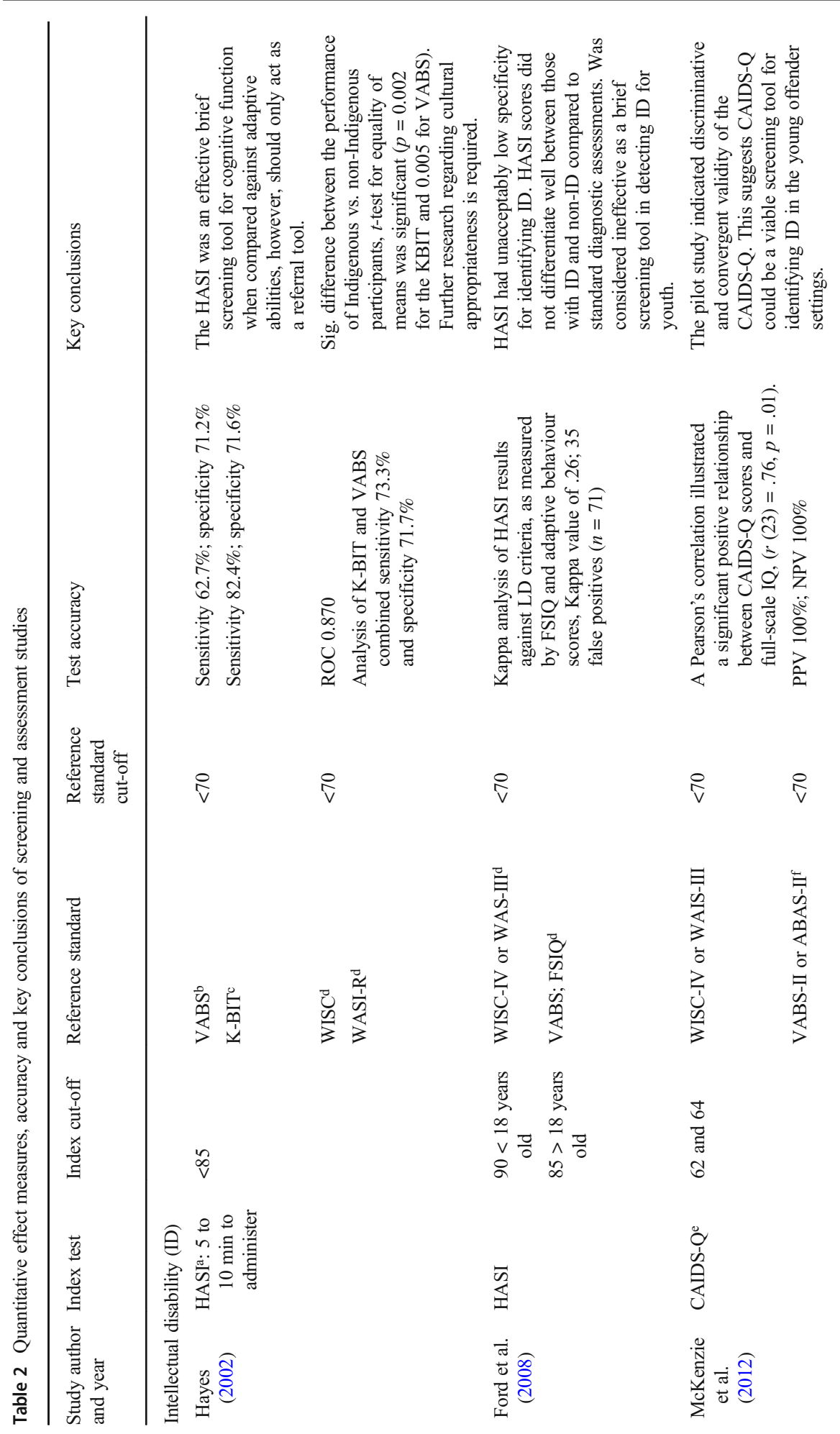




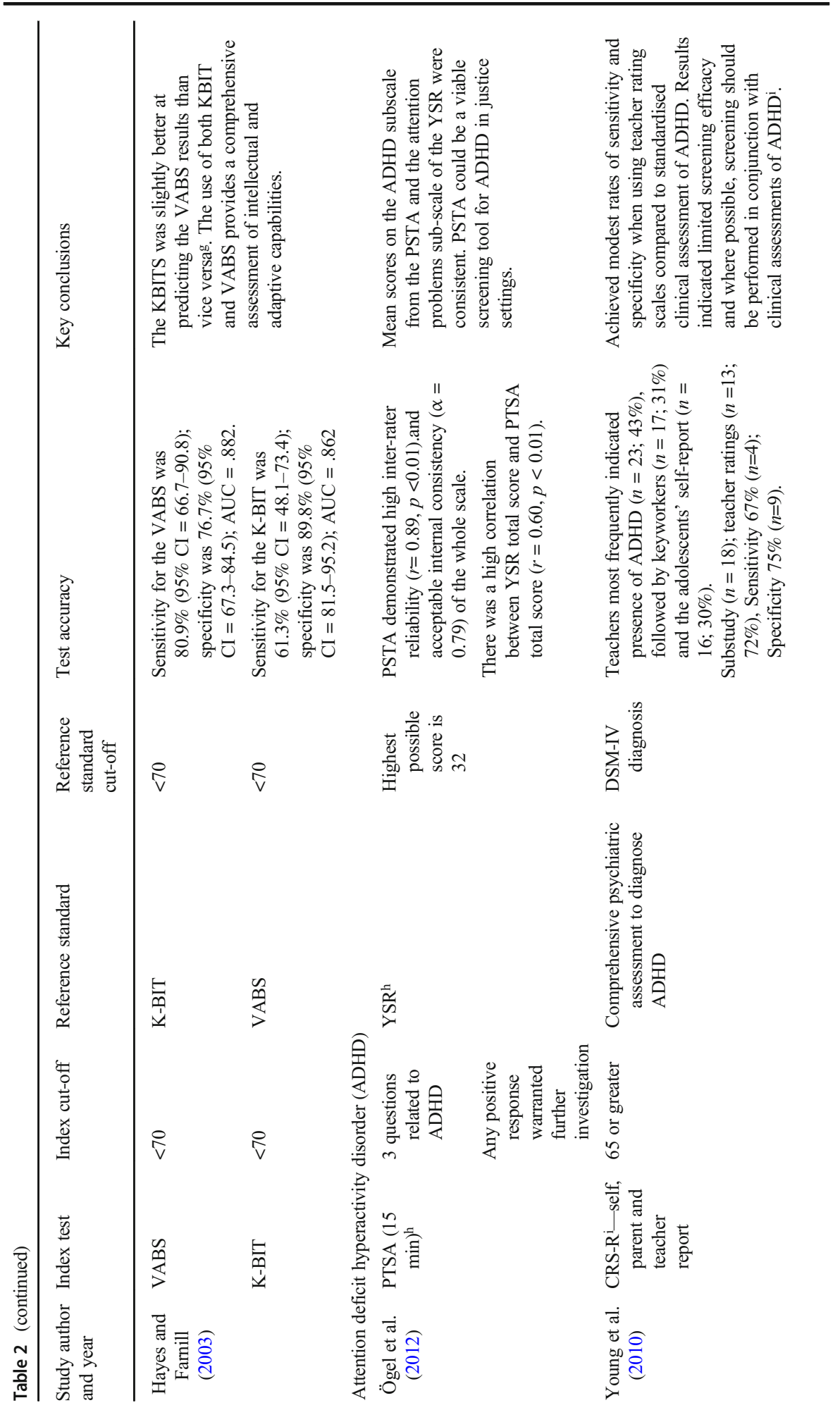




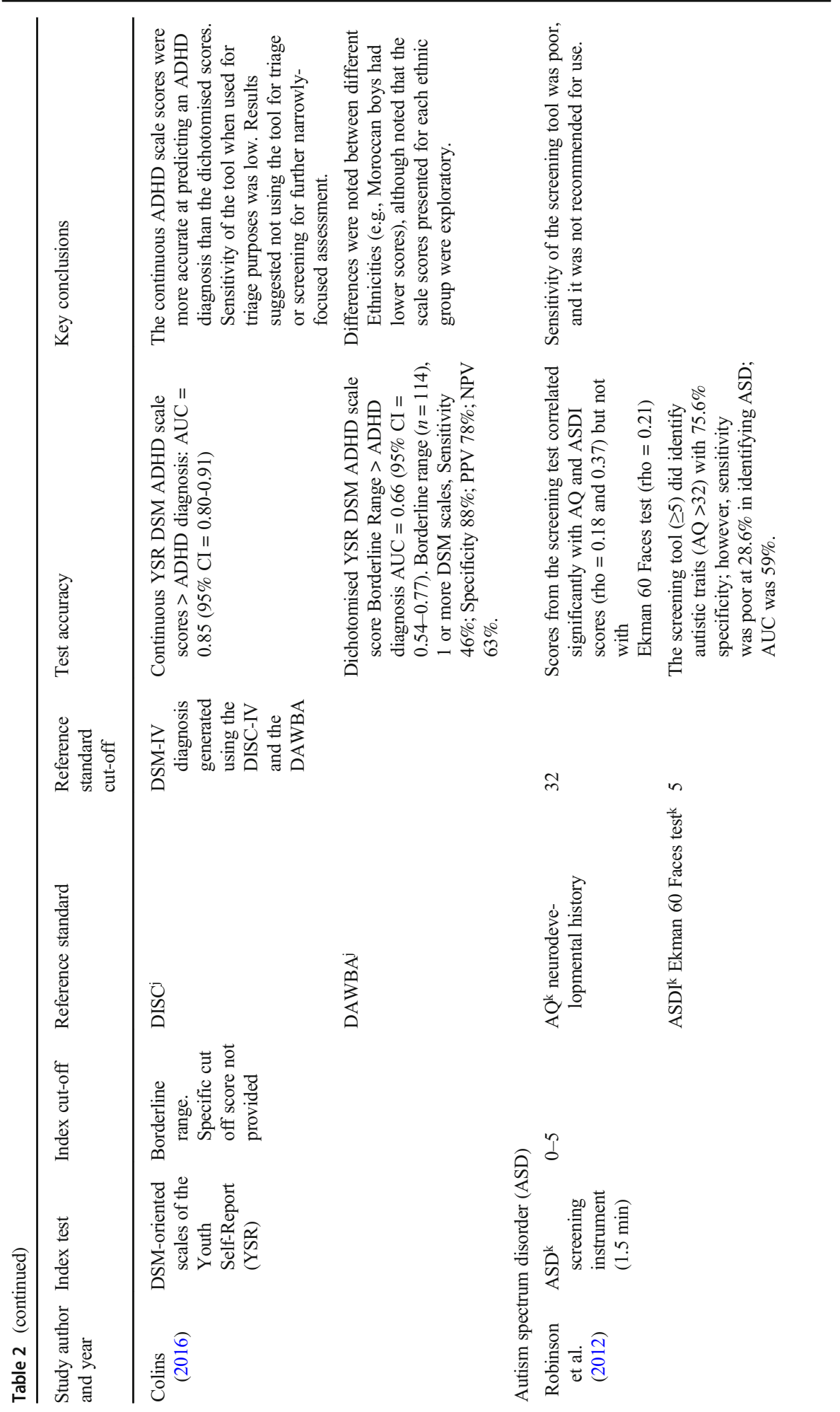




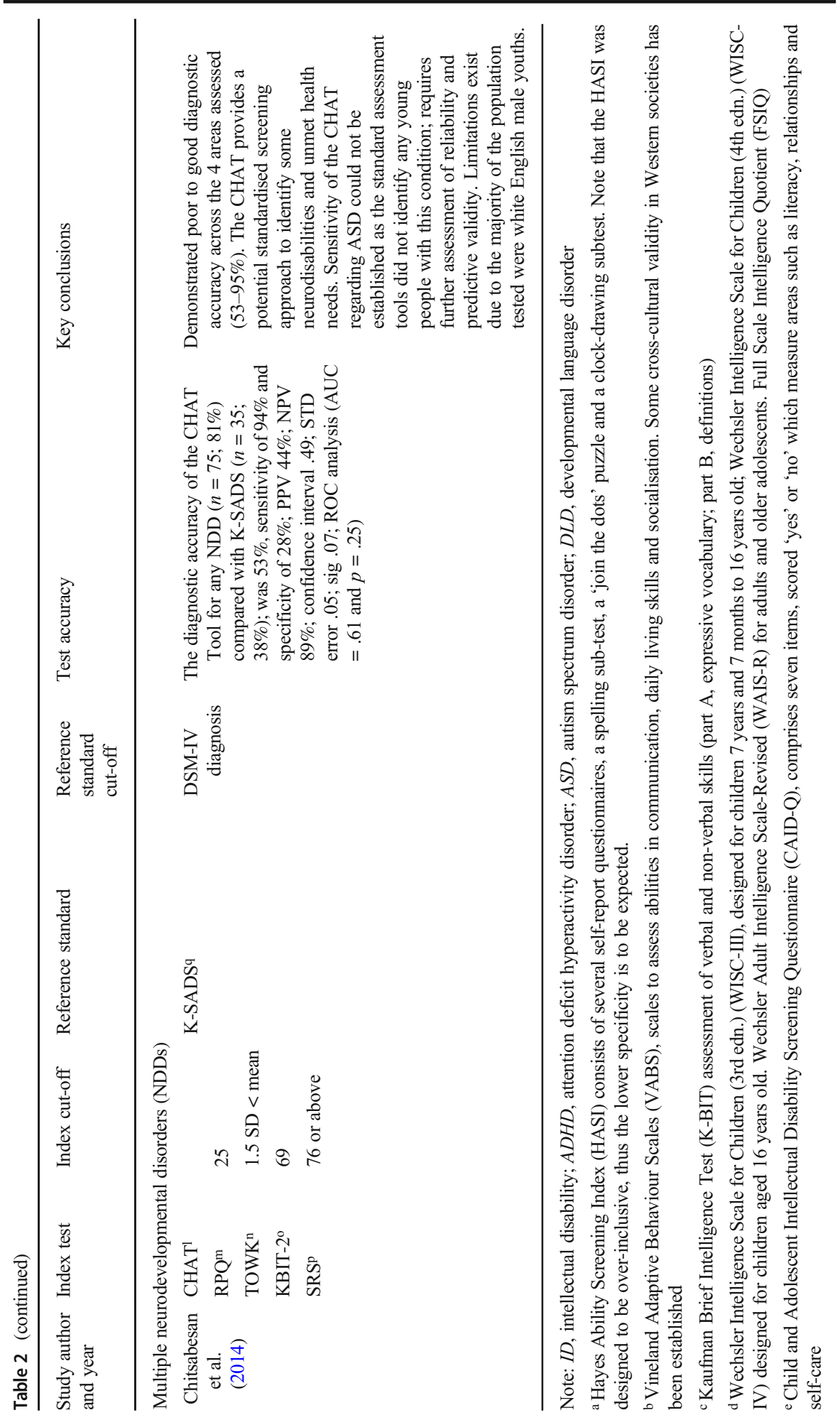




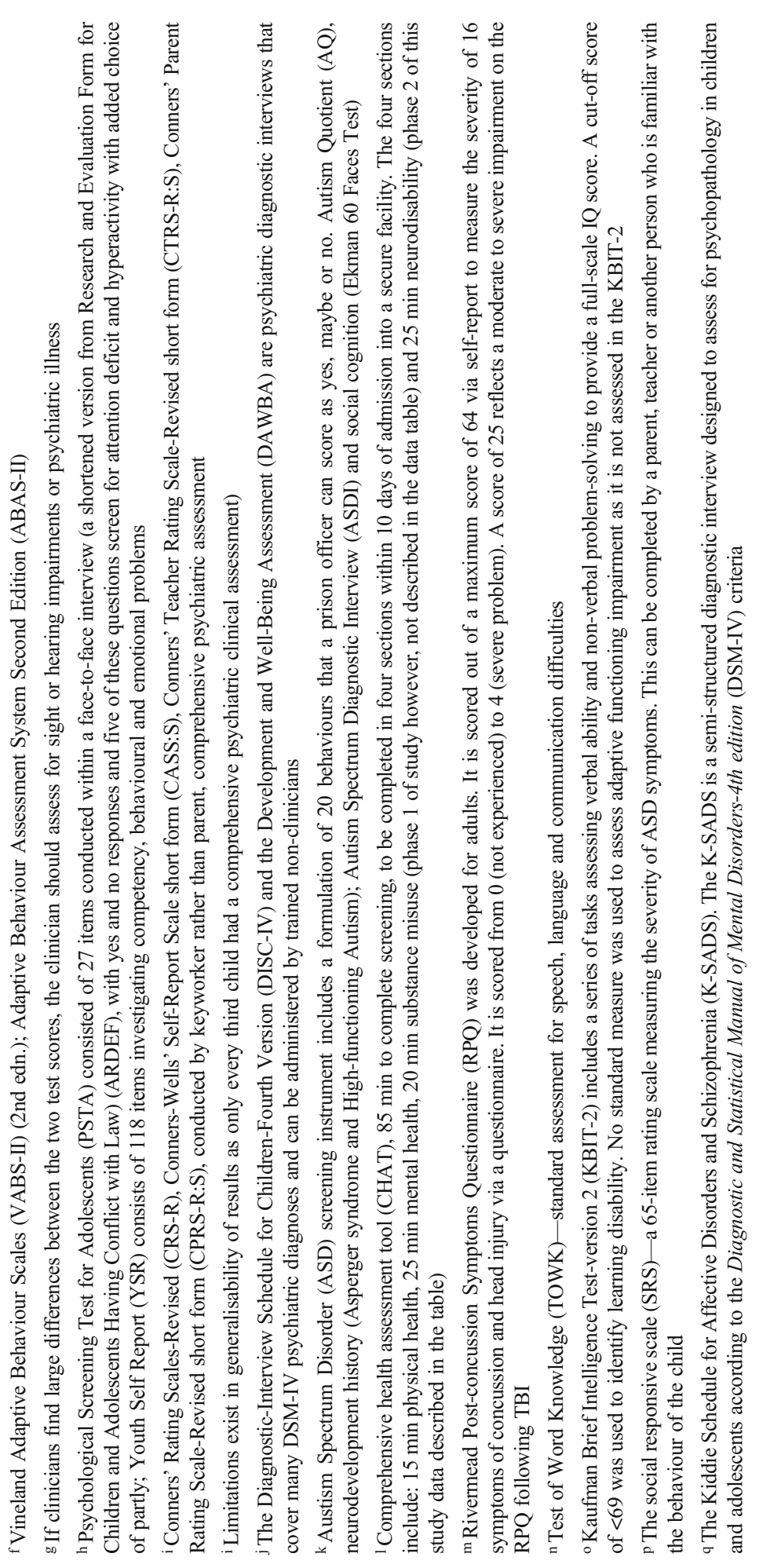




\section{Intellectual disability}

Four studies focused on screening and/or assessment for ID (Ford et al., 2008; Hayes and Farnill, 2003; Hayes, 2002; McKenzie et al., 2012). Two studies examined the Hayes Ability Screening Index (HASI) to screen for ID and had conflicting findings. Ford et al. (2008) found that the HASI may not be appropriate for detecting ID compared with the Wechsler Intelligence Scale for Children (3rd and 4th edn.; WISC-III/WAIS-IV) and Vineland Adaptive Behaviour Scales (VABS). Whereas Hayes (2002) found that compared with standardised assessments Kaufman Brief Intelligence Test (K-BIT) and the VABS, the HASI had high sensitivity and specificity. McKenzie et al. (2012) found that the Child and Adolescent Intellectual Disability Screening Questionnaire (CAIDS-Q) demonstrated discriminative validity and accurate prediction of ID, suggesting that the CAIDS-Q could be a valid screening tool. Hayes and Farnill (2003) compared scores on the K-BIT and VABS, finding that the K-BIT was slightly better at predicting the VABS results than vice versa. Notably, the mean scores for the K-BIT were slightly higher, which could indicate greater adaptive impairments compared to cognitive abilities. However, the VABS was completed as a self-report and therefore, may be influenced by the young person's level of awareness regarding their impairments.

\section{Attention deficit hyperactivity disorder}

Three studies were found for ADHD (Colins, 2016; Ögel et al., 2012; Young et al., 2010). Ögel et al. (2012) undertook a pilot study that found the Psychological Screening Test for Adolescents (PSTA) was a reliable tool, compared with the Youth Self Report (YSR) as the reference measure. In contrast, Colins (2016) used the YSR as a screening tool (as the index test) reliably identified ADHD, but was not considered an appropriate triage tool to screen for ADHD with co-existing other mental disorders when compared with the DiagnosticInterview Schedule for Children-Fourth Version (DISC-IV) and the Development and WellBeing Assessment (DAWBA) as the reference tools. Young et al. (2010) compared a comprehensive psychiatric assessment to the Conners' Rating Scales-Revised (CRS-R; completed by the young person, parent and teacher). Results indicated teachers provided the most valid screening compared with a comprehensive psychiatric assessment.

\section{Autism spectrum disorder}

One study was identified for ASD (Robinson et al., 2012). Scores from the screening test correlated significantly with Autism Quotient (AQ) and Autism Spectrum Diagnostic Interview (ASDI) scores, but not with Ekman 60 Faces test. Sensitivity of the screening tool was poor; therefore, this tool was not recommended for use.

\section{Screening for multiple conditions}

The CHAT screening tool examined by Chitsabesan et al. (2014) provided a potential standardised screening approach that assessed multiple conditions including: TBI, learning disability, ASD and DLD. Results for identification of other conditions, however, concluded that the CHAT required further assessment regarding the reliability 


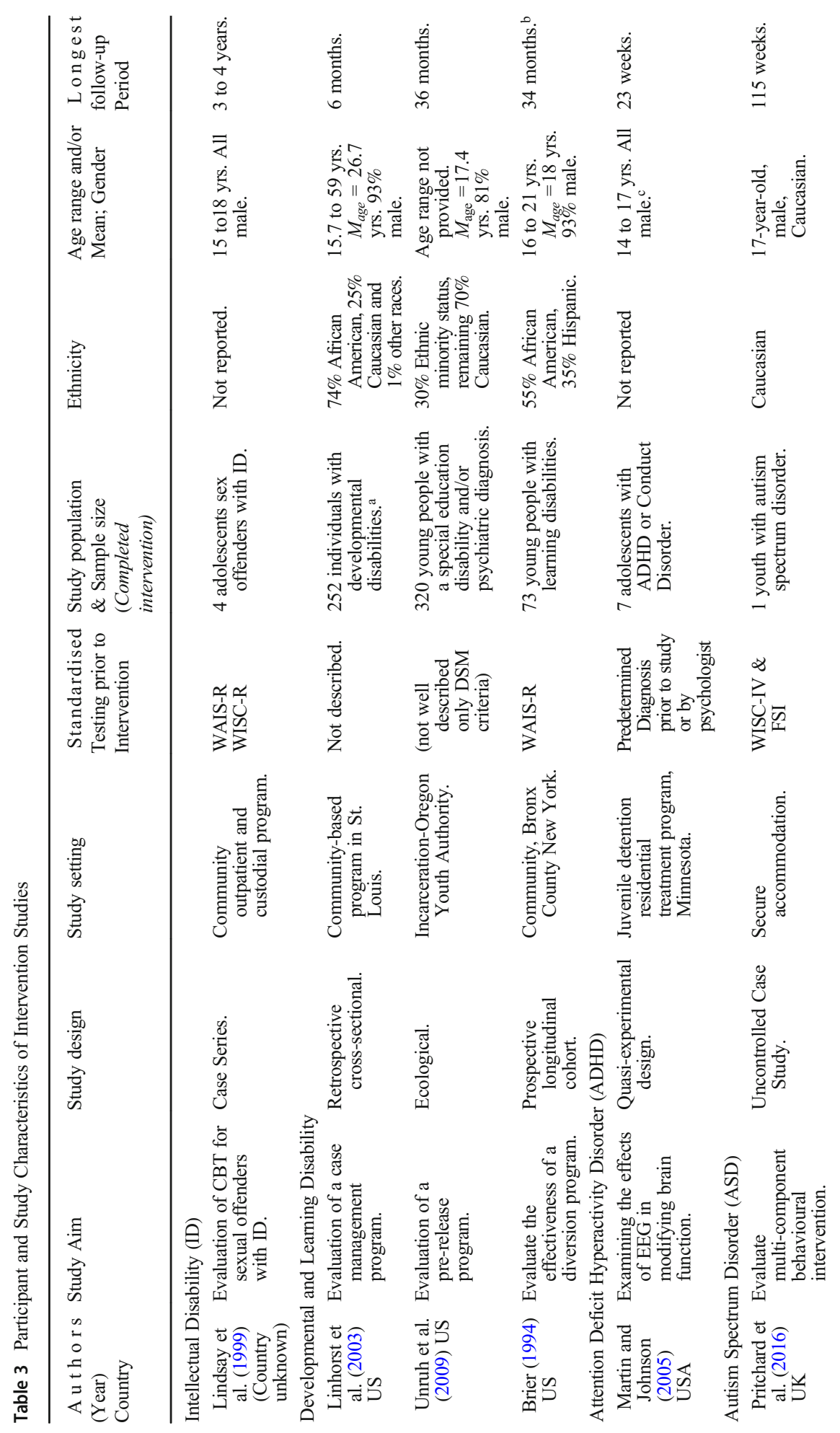




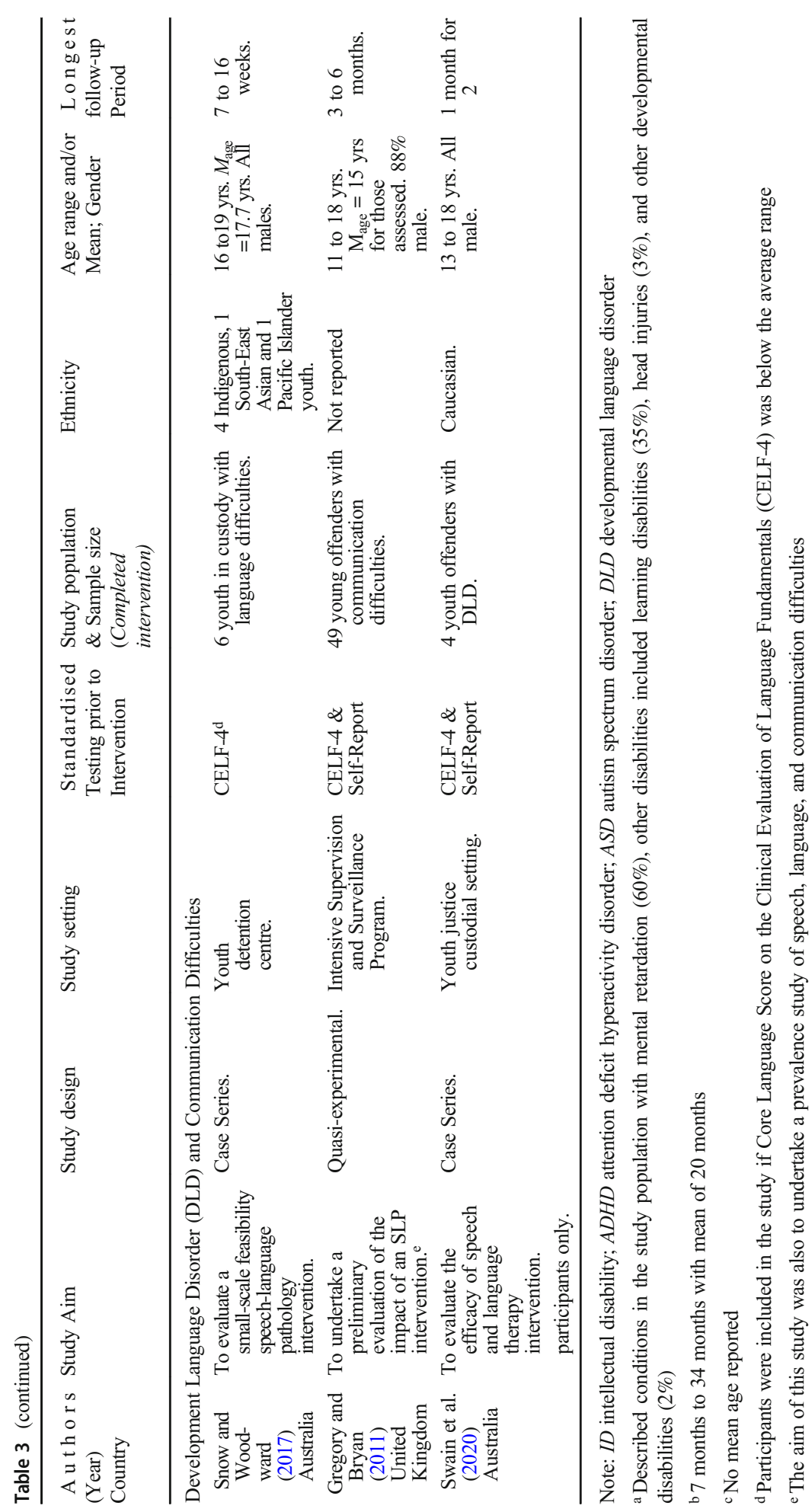


of detecting ASD, as no young people were included in the sample that had ASD. The results also described cultural limitations, specifically that most of the sample were described as white English male youth.

\section{Participant ethnicity and socioeconomic status for intervention studies}

Table 3 provides the participant characteristics for the intervention studies. Details of ethnicity were not included in three out of nine studies (Gregory and Bryan, 2011; Lindsay et al., 1999; Martin and Johnson, 2005). Four studies reported diverse ethnicities (Brier, 1994; Linhorst et al., 2003; Snow and Woodward, 2017; Unruh et al., 2009). Pritchard et al. (2016) described one youth participant of Caucasian heritage as did Swain et al. (2020). Only three studies comprehensively described social and demographic confounding variables, such as marital status, educational achievement, employment status, criminogenic risk factors and seriousness of crimes committed during follow-up periods (Brier, 1994; Linhorst et al., 2003; Unruh et al., 2009). Again, for the intervention studies, none of the included studies specifically addressed or focused on providing culturally appropriate intervention approaches for youth from First Nations backgrounds. Snow and Woodward (2017) did, however, recognise the need for culturally appropriate standardised language assessments to enhance pre-and post-assessment measures (despite the anticipated complexity of this), to determine the intervention outcomes of individualised speech and language therapy, for future research endeavours.

\section{Summary of intervention study outcomes}

Nine studies were included that provided interventions for youth with NDDs in the YJS (Table 4). Notably, most studies provided comprehensive standardised assessment by qualified health professionals prior to intervention. Intervention studies were diverse in their aims, approaches, theoretical frameworks and models to address needs of young people with NDDs. Key study outcomes included: improvements in communication and language skills, educational achievement, employment, living and social functioning; modification of criminogenic, dysfunctional or destructive behaviours; improved capacity to negotiate judicial processes; reductions in the negative impact of incarceration; reductions in recidivism; and improved quality of life for young people with a neurodevelopmental disorder exposed to the YJS.

\section{Intellectual disability}

One study evaluated a cognitive behaviour therapy (CBT) intervention for ID (Lindsay et al., 1999). This study found positive outcomes (e.g. development of empathy towards the victim, altered attitudes and cognitive distortions associated with sexual offenses toward children) for four offenders with ID that completed the CBT intervention.

\section{Developmental and learning disabilities}

Three studies were identified, which all aimed to reduce recidivism and offence rates (Brier, 1994; Linhorst et al., 2003; Unruh et al., 2009). Brier (1994) achieved 
a significant reduction in recidivism rates of $12 \%$ for youth with learning disabilities that completed the diversion program, versus $40 \%$ for non-completers and $38 \%$ for untreated controls. This study also achieved significant improvements in reading and arithmetic pre-to-post study. Similarly, Unruh et al. (2009) achieved a $15 \%$ recidivism rate for youth with special education disabilities and or psychiatric diagnoses compared to the $30 \%$ in the TRACS study, which was used as a control. Lastly, a community-based program to assist youth with developmental disabilities achieved significantly fewer arrests for those who completed the case management program compared to those who dropped out (25 and $43 \%$ respectively; Linhorst et al., 2003).

\section{Attention deficit hyperactivity disorder}

There was one study that evaluated 20 sessions of electroencephalogram (EEG) biofeedback in conjunction with treatment as usual for ADHD (Martin and Johnson, 2005). The study demonstrated $64 \%$ improved performance with seven adolescents with ADHD or conduct disorder in one or more standardised measures of sustained attention, response inhibition, intellectual ability and memory.

\section{Autism spectrum disorder}

One study reported improved quality of life for a youth with ASD through a multicomponent behaviour intervention (MCBI) program, which focused on the reduction of serious behavioural issues of aggression, absconding and inappropriate sexual behaviour (Pritchard et al., 2016). In total, it took 115 weeks of the intervention to integrate the youth safely back into mainstream education and supported community living.

\section{Developmental language disorder and communication difficulties}

Three studies that included evaluations of individualised speech-language pathology interventions reported positive results (Gregory and Bryan, 2011; Snow and Woodward, 2017; Swain et al., 2020). Gregory and Bryan (2011) achieved a 75\% improvement in targeted communication strategies and demonstrated $85-88 \%$ improvement in Clinical Evaluation of Language Fundamentals (CELF) subtests. Snow and Woodward (2017) indicated all participants demonstrated medium to high therapeutic engagement and five out of six demonstrated improvement on standardised measures at post-treatment. Swain et al. (2020) achieved medium-to-large treatment effects that were statistically significant in identifying changes to primary repeated measures of communication skills.

\section{Discussion}

Overall, the current review identified limited research that evaluated screening, assessment or intervention approaches for young people with neurodevelopmental disorders in the youth justice system. With regard to the screening or assessment studies, six out 


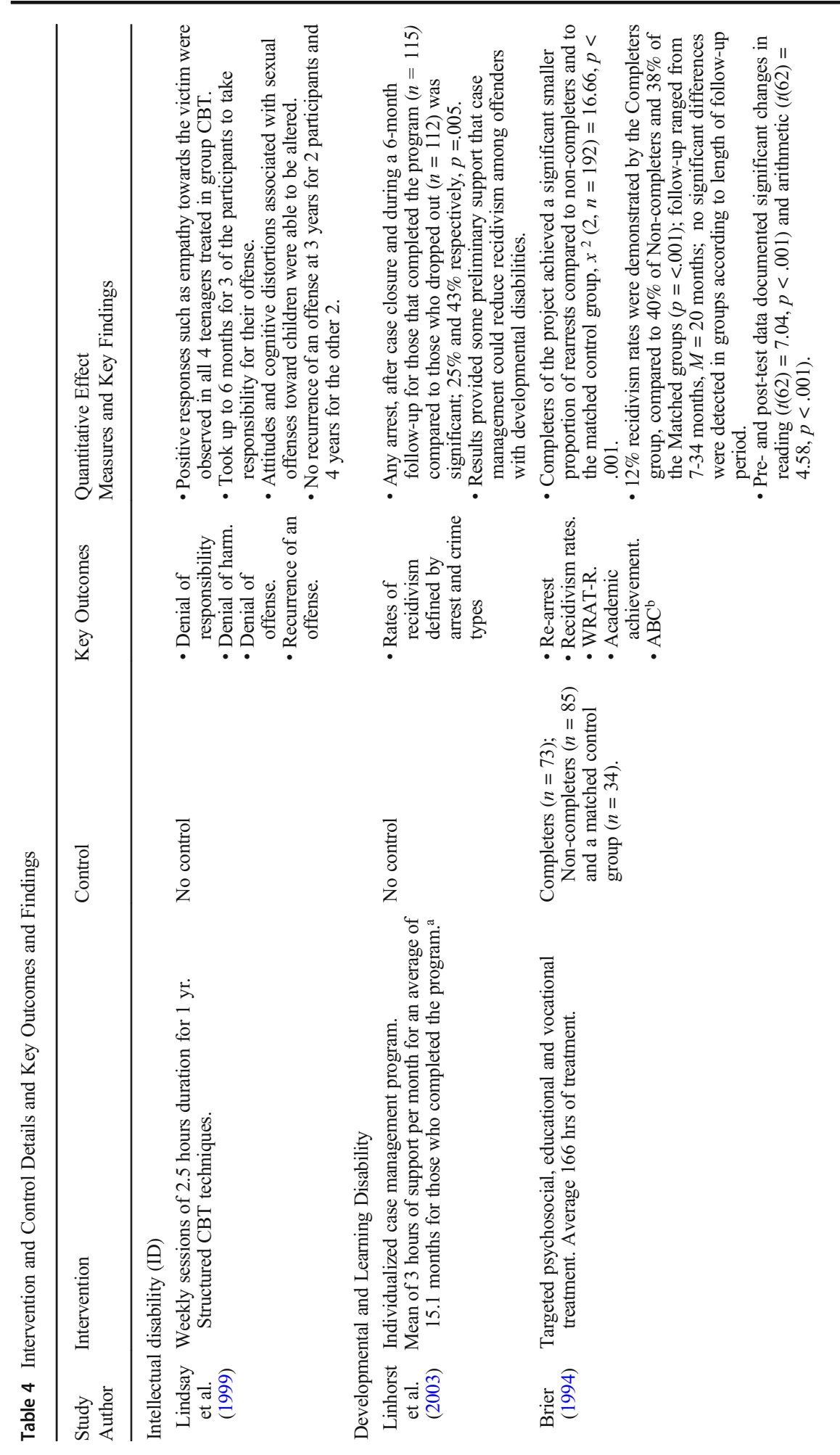




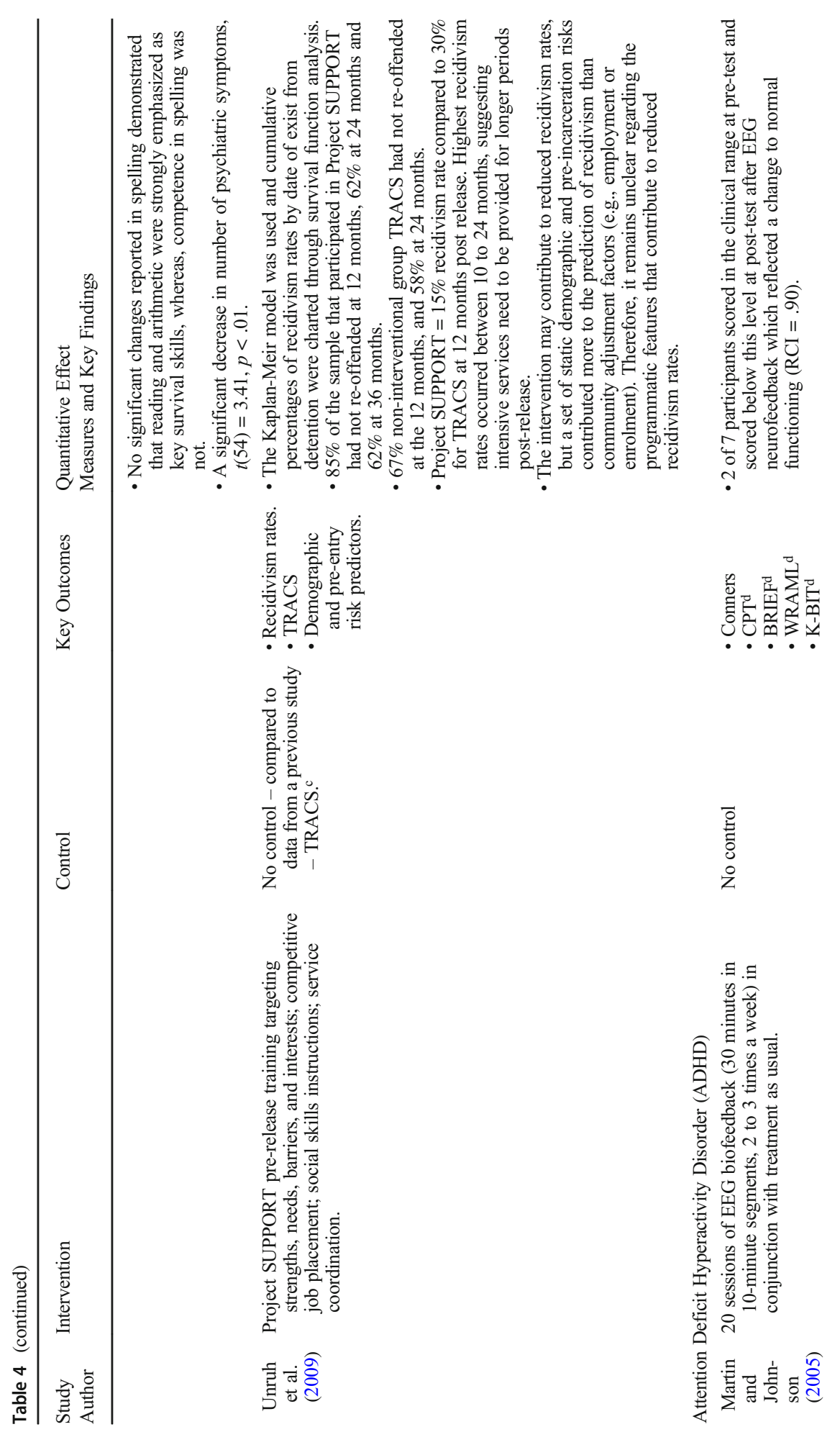




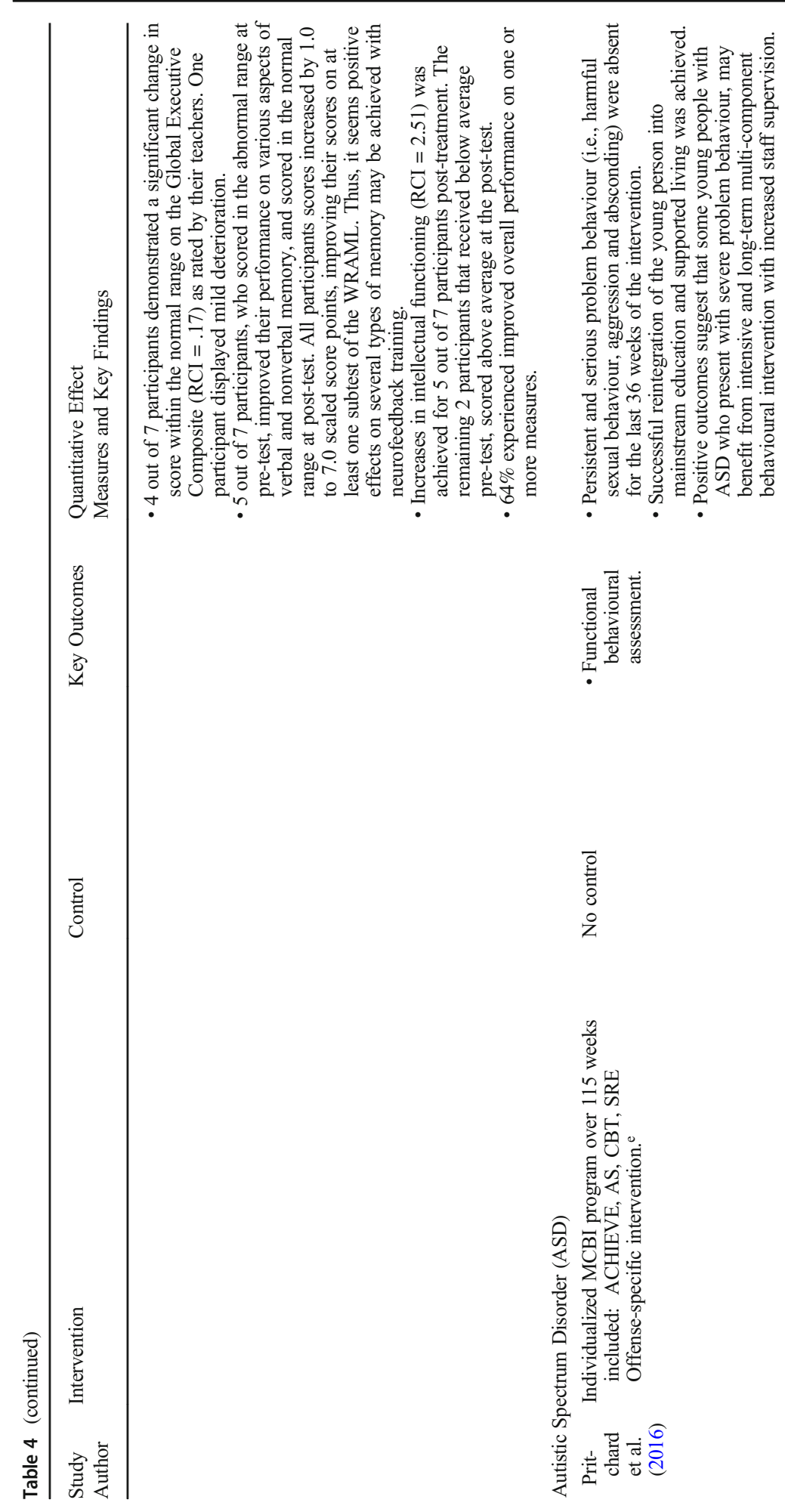




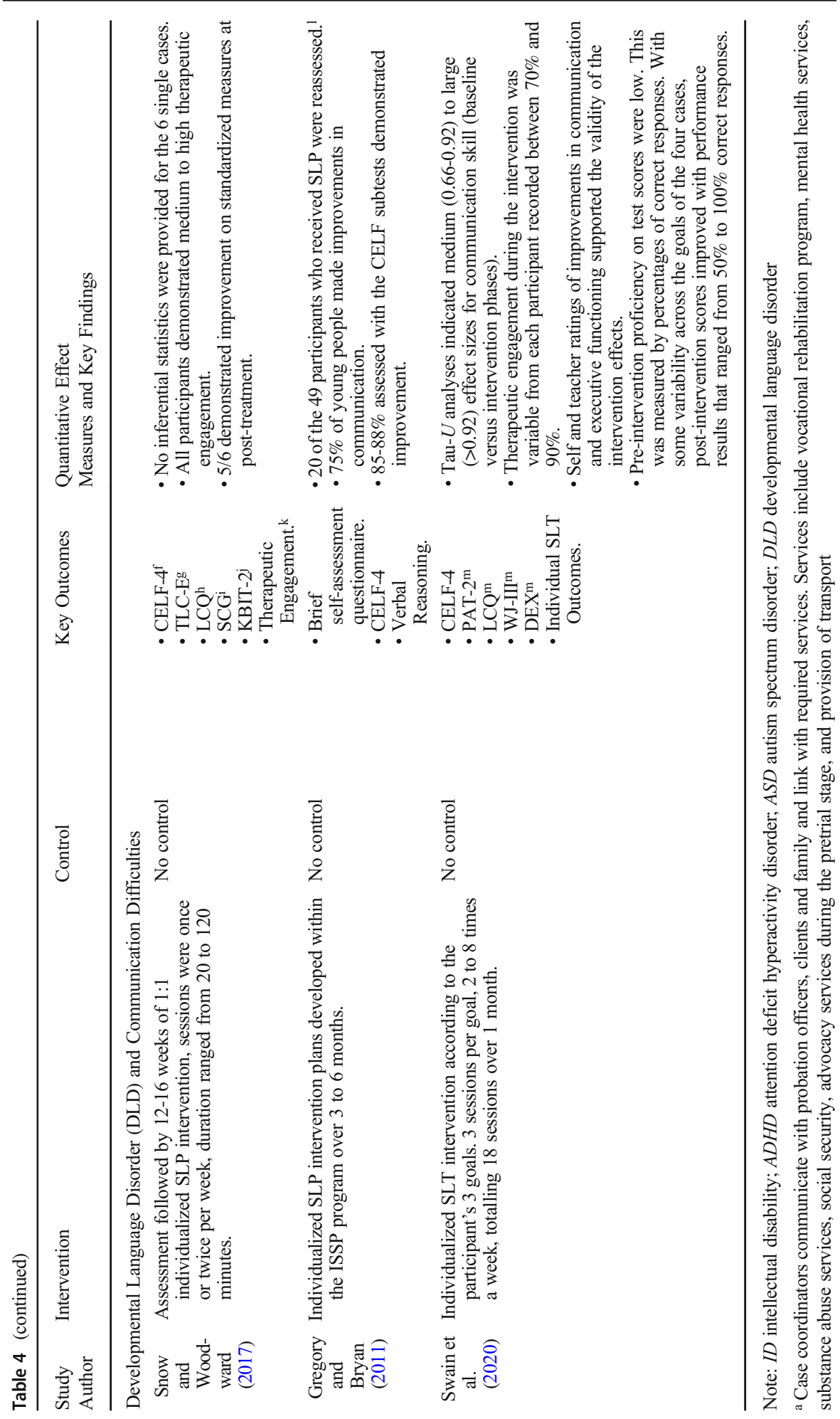




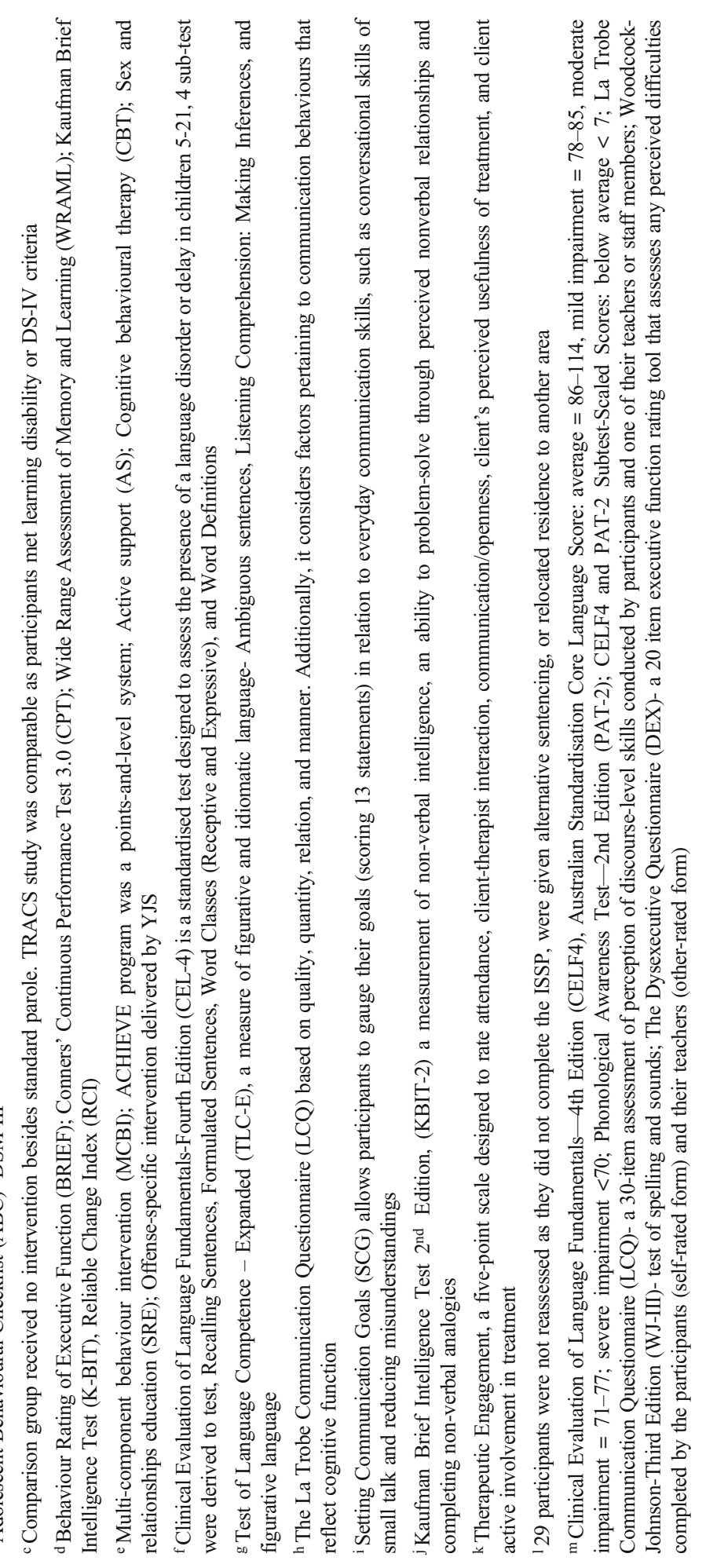


of nine screening and assessment tools were considered reliable in identifying NDDs in the YJS; however, the quality of these studies was generally poor. The three studies that were not reliable in identifying NDDs were focused on ASD (Robinson et al., 2012), ID (Ford et al., 2008) and multiple NDDs (Chitsabesan et al., 2014). In addition, Young et al. (2010) concluded that whilst the Conners' Rating Scales-Revised (CRS-R) may be of benefit to screen for the symptoms of ADHD, particularly when performed by teachers, it was important where possible that screening should be augmented by clinical assessments of ADHD. Furthermore, Colins (2016) found that the YSR was limited in accurately screening for ADHD alongside co-existing mental disorders and warns against the future use of similar narrowly focused assessment tools in the youth detention setting. Therefore, it is reasonable to propose that comprehensive and multidisciplinary standardised testing, conducted in a conducive environment, is required to reliably identify youth with NDDs in the YJS (Tinsley Li and Rogers, 2005). Notably, one protocol study was identified that included screening and/or assessment of FASD but did not implement or evaluate a program, and therefore did not meet inclusion criteria for this review (Passmore et al., 2016). This is of concern, given the prevalence and propensity for young people with FASD to become involved in the YJS (Bower et al., 2018).

Nine intervention studies were included in the current review. The interventions that had the greatest success were those that identified and prioritised participants' needs, goals, learning styles, strengths, skill deficits and environmental exposures to promote protective factors and reduce recidivism (Brier, 1994; Linhorst et al., 2003; Unruh et al., 2009). These interventions were best aligned with disabling critical criminology, which recognises the need for holistic, strength-based and patient-centred approaches. The interventions sought to understand the participants' requirements and addressed a range of theorised ecological factors concerning successful transition into adulthood and comprehensively addressed confounding environmental and societal impacts that contribute to crime risk and consequent detention (Dowse et al., 2009; DeKeseredy and Perry, 2006; Reid-Cunningham and Fleming, 2009; Baldry et al., 2018). Out of 22 ecological barriers assessed prior to re-entry into the community, Unruh (2009, p. 286) identified that the top three barriers were '(a) substance abuse (79\%), (b) absenteeism or suspension from school (79\%) and running away from home or residential placement (65\%)'. Additionally, two-thirds of participants had previously resided in foster care and had anger management issues.

Case management approaches, such as those exemplified in the studies by Unruh et al. (2009) and Linhorst et al. (2003) may provide a practical framework for supporting holistic, multidisciplinary and youth-centred interventions. In the Option for Justice for Persons with Developmental Disabilities (OFJ; Linhorst et al., 2003), case coordinators comprehensively assessed clients' needs and linked them to required resources by facilitating access to services and sharing information with these services. Similarly, 'Project SUPPORT' achieved a significant reduction in recidivism for youth with a special education disability, using a transition specialist who worked collaboratively with community agencies to support successful transition to the community postincarceration. This collaboration addressed engagement with school, employment, vocational and social interests, family connections, and living arrangements. Findings from this study suggest that intensive services need to be provided for longer periods 
post-release, and with greater follow-up of participants, as the highest recidivism rates occurred between 10 and 24 months (Unruh et al., 2009).

Additional studies demonstrated greater success when intensive interventions were provided over a longer time frame (Pritchard et al., 2016; Brier, 1994). Pritchard et al. (2016) provided a multi-component behavioural intervention conducted over 115 weeks. Dedicated staff documented and responded to targeted negative behaviours $24 \mathrm{~h}$ a day, 7 days a week and provided a reward system to positively reinforce good behaviour for a boy with ASD. This intervention entirely extinguished serious disruptive, absconding and inappropriate sexual behaviour. Additionally, Brier (1994) achieved significant reductions pre-to-post-program (after 34 months) in recidivism rates (12\% vs. $40 \%$ for non-completers and $38 \%$ untreated controls) and psychiatric symptoms $(p<.01)$, as well as improvement in reading $(p<.001)$ and arithmetic $(p<.001)$. The intervention delivered by Brier (1994) was a group diversion program that provided psychosocial, educational and vocational supports for youth with learning disabilities. This program involved two 90-min group sessions per week that averaged a total of $166.27 \mathrm{~h}$ of treatment at completion to achieve successful results (Brier, 1994).

It is important not to discount case series or case reports that provided intensive programs to improve the quality of life for youth with serious behavioural problems (Pritchard et al., 2016) and speech and language difficulties (Snow and Woodward, 2017; Swain et al., 2020). The study designs that were chosen may be appropriate when considering the complex needs of youth with NDDs, despite preferred designs such as RCTs that could enhance the quality of findings. Complex needs require individualised therapy plans, which enhance the provision of care and support optimal outcomes for consenting participants with NDDs in the YJS. The three programs offering individualised speech and language intervention had positive results according to post-test data with varying follow-up periods ranging from 7 to 16 weeks (Snow and Woodward, 2017), 3 to 6 months (Gregory and Bryan, 2011) and 4-8 weeks (Swain et al., 2020). Additionally, participants' self-reported satisfaction with the intervention and therapeutic engagement was observed, reflecting improvements in quality of life and language skills that could minimise recidivism risk (Swain et al., 2020; Snow and Woodward, 2017). Notably, there was an absence of intervention studies for some specific NDDs, including FASD and TBI. Although not meeting criteria for inclusion in the current review, Longstaffe et al. (2018) implemented a pilot program funded by Canada Justice, 'The Manitoba Youth Justice Program: Empowering and Supporting Youth with FASD in Conflict with the Law'. This program aimed to identify young people with FASD early in the judicial process; achieved by multi-disciplinary assessment designed to identify young people with FASD and provide appropriate support.

\section{Recommendations for future research and practice}

The current review revealed limited acknowledgement of cultural and linguistic diversity of young people and Indigenous-led approaches. This acknowledgement and consideration in service design and delivery is vital given the overrepresentation of Indigenous young people in youth justice settings globally. 
Researchers need to proceed cautiously to ensure inclusivity of Indigenous peoples in decision making, in a manner that values cultural training of staff, respects an inclusive approach of linguistic and cultural diversity and is committed to the delivery of culturally capable services which can provide appropriate alternatives to youth detention for young people with a NDD (Gilman et al., 2015; Hamilton, Reibel, et al., 2020; Human Rights Watch, 2018; Kippin et al., 2018). Only four out of the nine screening and assessment studies considered the cultural and linguistic backgrounds of the participants (Hayes, 2002; Young et al., 2010; Chitsabesan et al., 2014; Colins, 2016). One possible consequence of this is that a young person's performance on tests may be reduced (Durand and Christodulu, 2005), which may lead to erroneous labelling of unfit for trial, precluding adequate support to enable a rigorous defence (McSherry et al., 2017). Additionally, six out of nine of the intervention studies provided demographic details of youth's cultural and linguistic backgrounds. However, cultural capability was not adequately considered in the design of these intervention studies.

Indigenous young people with NDDs in the YJS require strength-based approaches (Hamilton, Maslen, et al., 2020). Future research requires Indigenous-led ways of knowing, of being and of doing, to support beneficial interventions for individuals and the community that maintain resistance against racialising practices and negative stereotypical labelling of Indigenous people (Askew et al., 2020; Bond and Singh, 2020; Toombs, 2012). Hamilton, Maslen, et al. (2020) describes an exemplar of therapeutic future-focused assessment alongside a prevalence study (utilising standardised multidisciplinary assessment) of Indigenous youth via a 'recovery capital' model; perspectives from incarcerated youth with a NDD were heard through culturally safe social yarning practices and empathic listening, and support was offered for youth who had NDDs and communication difficulties (Hamilton, Maslen, et al., 2020). This process respects a young person's participation in decision making and validates the self-expression of their assets and values regarding relationships, connections to family, culture, country, enjoyed social activities and community supports, and their educational, vocational and tertiary education ambitions (Hamilton, Maslen, et al., 2020).

Both disabling critical criminology (Dowse et al., 2009; Baldry et al., 2018) and abolitionist perspectives (Brown and Schept, 2017; Goldson, 2005) highlight the urgent need for policy and legislative changes enacting the compulsory provision of effective screening and assessment during early years of life to detect NDDs and establish appropriate interventions. These interventions (i.e. health, educational, speech and language and behavioural treatments) are essential for enabling improved life trajectories and prevention of youth detention (Hopkins et al., 2018). In addition, inclusive community infrastructure that establishes equal access to education, employment and wealth creation can promote social and emotional wellbeing of children globally, thereby reducing criminogenic risk and youth detention rates (Baldry et al., 2018; McCausland and Baldry, 2018; DeKeseredy and Perry, 2006; Reid-Cunningham and Fleming, 2009). It is imperative to reduce the funnelling of young people with multifaceted complex needs into lifelong adult incarceration by addressing the interconnecting issues of NDDs, health and educational needs, social disadvantage and exclusion and criminogenic risk within childhood (Baldry et al., 2018). Therefore, to counter this situation a collaborative and intersectoral policy shift is required, in which greater funding is allocated towards prevention strategies that eliminate the 
incarceration and reduce the recidivism of young people with NDDs (Hamilton, Reibel, et al., 2020; Lambie 2020; Passmore et al., 2018; Brown and Schept, 2017; Goldson, 2005). Prevention strategies require an interdisciplinary and holistic care approach, via government action and policy change that directs responses that are proportionately delivered according to disadvantage and disability (Baldry et al. 2017).

\section{Strengths and limitations}

The current systematic review has provided the first comprehensive overview of three areas of need within the YJS for young people with NDDs - screening, assessment and intervention. However, a number of limitations need to be considered, including the potential effects of publication and/or non-reporting bias (Page et al., 2021). There were no published protocols or publicly available (e.g. open science platform) documents for the included studies. Future research would benefit from published protocols or uploading study protocol documents to available public sites. Additionally, the exclusion of grey literature heightens the risk of biased reporting within the current systematic review as unpublished or unevaluated screening, assessment and interventions have not been discussed (Ahmed et al., 2012). Further, only English-language publications were included, and most studies were conducted in First World nations.

Additionally, a limitation within the literature more broadly is a lack of consideration of NDDs in a wide range of studies, not eligible for the current review. Many studies (e.g. Brugman and Bink, 2011; Currie et al., 2012; Lennox et al., 2015), examined the effectiveness of screening and interventions for conditions or problems that may co-occur with NDDs, such as aggression, mental health disorders, delinquency, substance use disorders and social criminogenic risks but did not assess or report on the presence of NDDs. This may limit the recognition and understanding of potential differential treatment effects and diminish broader understanding of the complex issues and co-occurring conditions facing young people with NDDs in YJS settings and the need to address these issues holistically. A further finding that may bias results within most of the studies was the lack of analysis of contextual confounding variables; findings revealed a lack of consideration for criminogenic risk, coexisting social disadvantage, and a young person's access to education, future employment opportunities, and community and health services. Appropriate identification of NDDs and diversion strategies to prevent incarceration or recidivism will only be achieved by addressing coexisting social gradient issues, structural racism and discriminatory practices that contribute to the harm and trauma experienced by marginalised and vulnerable groups of young people.

\section{Conclusion}

The current systematic review provides a narrative synthesis of the available evidence regarding screening, assessment and interventions for young people with neurodevelopmental disorders who are involved with the youth justice system. The review highlights significant gaps in research to effectively address complex needs and counter the disproportionate representation of youth with NDDs in the YJS. Therefore, it supports the need for further research and associated reform to improve identification and care of young people with NDDs who are exposed to the YJS. Collectively as 
researchers, advocates and responsive global citizens it is time to question established legal environments that normalise the processing and incarceration of young people with NDDs. It is morally and ethically irresponsible to ignore an atmosphere in which the control, punitive management and punishment of youth with disabilities may be accepted and the provision of appropriate programs may be deficient. Detaining youth with a NDD violates the fundamental notion of a child's right to freedom, liberty and protection from harm. This systematic review highlights the need for global attention regarding young people with NDDs at risk of YJS involvement and the issues they face in these settings.

Supplementary Information The online version contains supplementary material available at https:/doi.org/ 10.1007/s11292-021-09475-w.

Acknowledgements I would like to acknowledge the Turrbul and Jagera people as the traditional owners and custodians of the land in which this work was conducted. On behalf of The University of Queensland (UQ) I would like to pay our respects to their Ancestors and their descendants, who continue cultural and spiritual connections to Country. We recognise their valuable contributions to Australian and global society. I would like to sincerely thank my academic supervisors and co-authors Dr. Andrew Smirnov and Dr. Natasha Reid for their dedication, diligence and excellent research standards and integrity in mentoring me through the rigorous processes of a systematic review. I would like to acknowledge and thank UQ librarians Felicity Berends and Scott Macintyre for their guidance and education whilst undertaking this research project. I would like to acknowledge and thank Dr. Danay Baker-Andresen from the UQ Aboriginal and Torres Strait Islander Studies Unit and Dr. Debby Lynch from the UQ School of Nursing, Midwifery and Social Work for being willing to critically read and provide valuable feedback concerning this manuscript. I am also very grateful for the expertise gained by senior biostatistician Associate Professor Federica Bazri, from the UQ Poche Centre who reviewed our quantitative data analysis. Lastly, thank you to my dedicated husband John, my beautiful family and friends for believing in me and supporting my dreams of pursuing academic excellence.

Statement of authorship Mrs Holland conceptualised and designed the study, drafted the initial manuscript, completed manuscript revisions and approved the final manuscript as submitted. Dr Reid conceptualised and designed the study, critically reviewed and revised the manuscript and approved the final manuscript as submitted. Dr Smirnov conceptualised and designed the study, critically reviewed and revised the manuscript and approved the final manuscript as submitted.

Funding This research did not receive any specific grant from funding agencies in the public, commercial or not-for-profit sectors.

\section{Declarations}

\section{Declarations of interests None.}

Open Access This article is licensed under a Creative Commons Attribution 4.0 International License, which permits use, sharing, adaptation, distribution and reproduction in any medium or format, as long as you give appropriate credit to the original author(s) and the source, provide a link to the Creative Commons licence, and indicate if changes were made. The images or other third party material in this article are included in the article's Creative Commons licence, unless indicated otherwise in a credit line to the material. If material is not included in the article's Creative Commons licence and your intended use is not permitted by statutory regulation or exceeds the permitted use, you will need to obtain permission directly from the copyright holder. To view a copy of this licence, visit http://creativecommons.org/licenses/by/4.0/. 


\section{References}

Abramovitz, R., \& Mingus, J. (2016). Unpacking racism, poverty, and trauma's impact on the school-toprison pipeline. In A. Carten, A. Siskind, \& M. Pender Greene (Eds.), Strategies for deconstructing racism in the health and human services (pp. 245-265). Oxford University Press, Incorporated.

Ahmed, I., Sutton, A. J., \& Riley, R. D. (2012). Assessment of publication bias, selection bias, and unavailable data in meta-analyses using individual participant data: a database survey. BMJ, 344, 16-16. https://doi. org/10.1136/bmj.d7762.

Akers, R. L., Sellers, C. S., \& Jennings, W. G. (2017). Criminological theories: Introduction, evaluation, and application. Seventh edition. Oxford University Press.

Allen, J., Balfour, R., Bell, R., \& Marmot, M. (2014). Social determinants of mental health. International Review of Psychiatry: Public Mental Health, 26, 392-407. https://doi.org/10.3109/09540261.2014. 928270.

American Psychiatric Association. (2013). Neurodevelopmental disorders. In Diagnostic and statistical manual of mental disorders: DSM-5. (Fifth edition.) (Section 2). Arlington, VA: American Psychiatric Association.

Arthur, R. (2016). The moral foundations of the youth justice system: Understanding the principles of the youth justice system. Routledge.

Askew, D., Brady, K., Mukandi, B., Singh, D., Sinha, T., Brough, M., \& Bond, C. J. (2020). Closing the gap between rhetoric and practice in strengths-based approaches to Indigenous public health: a qualitative study. Australian and New Zealand Journal of Public Health, 44, 102-105. https://doi.org/10.1111/17536405.12953.

Australian Institute of Health and Welfare. (2018) Youth detention population in Australia 2018. Retrieved July 26, 2019, from AIHW, Canberra, Australian Government Web site: https://www.aihw.gov.au/ reports/youth-justice/youth-detention-population-in-australia-2018/data

Bailey, Z. D., Krieger, N., Agénor, M., Graves, J., Linos, N., \& Bassett, M. T. (2017). Structural racism and health inequities in the USA: Evidence and interventions. The Lancet, 389, 1453-1463. https://doi.org/10. 1016/S0140-6736(17)30569-X.

Baird, G. (2008). Assessment and investigation of children with developmental language disorder. In C. Norbury, J. B. Tomblin, \& D. V. M. Bishop (Eds.), Understanding developmental language disorders: From theory to practice (pp. 1-22). Psychology Press Taylor \& Francis Group.

Baker, J. (2017). The duty we owe: Foetal alcohol spectrum disorder, Indigenous imprisonment and Churnside V Western Australia [2016] WASCA 146. University of Western Australia Law Review, 42, 119-135.

Baldry, E., Briggs, D. B., Goldson, B., \& Russell, S. (2018). 'Cruel and unusual punishment': An interjurisdictional study of the criminalisation of young people with complex support needs. Journal of Youth Studies, 21, 636-652. https://doi.org/10.1080/13676261.2017.1406072.

Bland, M. (2015). An introduction to medical statistics (4th ed.). Oxford University Press.

Bond, C. J., \& Singh, D. (2020). More than a refresh required for closing the gap of indigenous health inequality. Medical Journal of Australia, 212, 198-199. https://doi.org/10.5694/mja2.50498.

Bower, C. \& Elliott E. (2016). On behalf of the steering committee: Report to the Australian government of health: Australian guide to the diagnosis of fetal alcohol spectrum disorder (FASD). Retrieved August 15, 2019, funded by Australian Government, Department of Health Website: https://www.fasdhub.org.au/ siteassets/pdfs/australian-guide-to-diagnosis-of-fasd_all-appendices.pdf

Bower, C., Watkins, R. E., Mutch, R. C., Marriott, R., Freeman, J., Kippin, N. R., Safe, B., Pestell, C., Cheung, C. S. C., Shield, H., Tarratt, L., Springall, A., Taylor, J., Walker, N., Argiro, E., Leitão, S., Hamilton, S., Condon, C., Passmore, H. M., \& Giglia, R. (2018). Fetal alcohol spectrum disorder and youth justice: A prevalence study among young people sentenced to detention in Western Australia. British Medical Journal Open, 8(2), e019605 Retrieved 19 July 2019 from https://bmjopen.bmj.com/ content/8/2/e019605.

Brier, N. (1994). Targeted treatment for adjudicated youth with learning disabilities: Effects on recidivism. Journal of Learning Disability, 27, 215-222. https://doi.org/10.1177/002221949402700403.

Brown, M., \& Schept, J. (2017). New abolition, criminology and a critical carceral studies. Punishment \& Society, 19, 440-462. https://doi.org/10.1177/1462474516666281.

Brugman, D., \& Bink, M. D. (2011). Effects of the EQUIP peer intervention program on self-serving cognitive distortions and recidivism among delinquent male adolescents. Psychology, Crime \& Law, 17, 345-358. https://doi.org/10.1080/10683160903257934. 
Burd, L., Fast, D. K., Conry, J., \& Williams, A. (2010). Fetal alcohol spectrum disorder as a marker for increased risk of involvement with correction systems. Journal of Psychiatry and Law, 38, 559-583. https://doi.org/10.1177/009318531003800408.

Chitsabesan, P., Lennox, C., Theodosiou, L., Law, H., Bailey, S., \& Shaw, J. (2014). The development of the comprehensive health assessment tool for young offenders within the secure estate. The Journal of Forensic Psychiatry and Psychology, 25, 1-25. https://doi.org/10.1080/14789949.2014.882387.

Colins, O. F. (2016). Psychometric properties and clinical usefulness of the youth self-report DSM-oriented scales: A field study among detained male adolescents. International Journal of Environmental Research and Public Health, 13, 932-945. https://doi.org/10.3390/ijerph13090932.

Currie, M. R., Wood, C. E., Williams, B., \& Bates, G. W. (2012). Aggression replacement training (ART) in Australia: A longitudinal youth justice evaluation. Psychiatry, Psychology and Law, 19, 577-604. https:// doi.org/10.1080/13218719.2011.615807.

DeKeseredy, W., \& Perry, B. (2006). Advancing critical criminology: Theory and application. Critical perspectives on crime and inequality. Lexington Books.

Downs, S. H., \& Black, N. (1998). The feasibility of creating a checklist for the assessment of the methodological quality both of randomised and non-randomised studies of health care interventions. Journal of Epidemiology and Community Health, 52, 377-384. https://doi.org/10.1136/jech.52.6.377.

Dowse, L., Baldry, E., \& Snoyman, P. (2009). Disabling criminology: Conceptualising the intersections of critical disability studies and critical criminology for people with mental health and cognitive disabilities in the criminal justice system. Australian Journal of Human Rights, 15(1), 29-46. https://doi.org/10.1080/ 1323238X.2009.11910860.

Durand, V. M., \& Christodulu, K. V. (2005). Mental retardation. In M. Hersen (Ed.), Clinician's handbook of child behavioural assessment (pp. 459-472). Elsevier Science \& Technology.

Fitzgerald, A. J. (2019). Ko te rongoa, ko te aro, ko te whai kia tika ai, mo nga rangatahi, solution-focused justice for young people. Paper presented 2019 University of Queensland Brisbane workshop: How do we advance interagency solution-focused approaches in Queensland, Queensland Children's Hospital, Brisbane, QLD, Australia

Ford, G., Andrews, R., Booth, A., Dibdin, J., Hardingham, S., \& Kelly, T. (2008). Screening for learning disability in an adolescent forensic population. Journal of Forensic Psychiatry and Psychology, 19, 371381. https://doi.org/10.1080/14789940802120944.

Ford, J., \& Blaustein, M. (2013). Systemic self-regulation: A framework for trauma-informed services in residential juvenile justice programs. Journal of Family Violence, 28, 665-677. https://doi.org/10.1007/ s10896-013-9538-5.

Gilman, A., Hill, K., \& Hawkins, J. (2015). When is a youth's debt to society paid? Examining the long-term consequences of juvenile incarceration for adult functioning. Journal of Developmental and Life-Course Criminology, 1, 33-47. https://doi.org/10.1007/s40865-015-0002-5.

Goldson, B. (2005). Child imprisonment: a case for abolition. Youth Justice, 5, 77-90. https://doi.org/10.1177/ 147322540500500202.

Greenhalgh, T., Thorne, S., \& Malterud, K. (2018). Time to challenge the spurious hierarchy of systematic over narrative reviews? European Journal of Clinical Investigation. https://doi.org/10.1111/eci.12931.

Gregory, J., \& Bryan, K. (2011). Speech and language therapy intervention with a group of persistent and prolific young offenders in a non-custodial setting with previously undiagnosed speech, language and communication difficulties. International Journal of Language and Communication Disorders, 46, 202215. https://doi.org/10.3109/13682822.2010.490573.

Hamilton, S. L., Maslen, S., Best, D., Freeman, J., O'Donnell, M., Reibel, T., Mutch, R., \& Watkins, R. (2020). Putting 'justice' in recovery capital: Yarning about hopes and futures with young people in detention. International Journal for Crime, Justice and Social Democracy, 9, 20-36. https://doi.org/10. 5204/ijcjsd.v9i2.1256.

Hamilton, S., Reibel, T., Maslen, S., Watkins, R., Freeman, J., Passmore, H., Mutch, R., O’Donnell, M., Braithwaite, V., \& Bower, C. (2020). Disability "in-justice": The benefits and challenges of "yarning" with young people undergoing diagnostic assessment for fetal alcohol spectrum disorder in a youth detention center. Qualitative Health Research, 30, 314-327. https://doi.org/10.1177/1049732319882910.

Hayes, S. C. (2002). Early intervention or early incarceration? Using a screening test for intellectual disability in the criminal justice system. Journal of Applied Research in Intellectual Disabilities, 15, 120-128. https://doi.org/10.1046/j.1468-3148.2002.00113.x.

Hayes, S. C., \& Farnill, D. (2003). Correlations for the vineland adaptive behavior scales with Kaufman brief intelligence test in a forensic sample. Psychology Reports, 92, 573-580. https://doi.org/10.2466/pr0.2003. 92.2.573. 
Hopkins, T., Clegg, J., \& Stackhouse, J. (2018). Examining the association between language, expository discourse and offending behaviour: an investigation of direction, strength and independence. International Journal of Language and Communication Disorders, 53, 113-129. https://doi.org/10. 1111/1460-6984.12330.

Hughes, N., Sheahan, F., Williams, W. H., \& Chitsabesan, P. (2020). Ensuring the rights of children with neurodevelopmental disabilities within child justice systems. The Lancet Child and Adolescent Health, 4, 163-166. https://doi.org/10.1016/S2352-4642(19)30401-8.

Hughes, N., Williams, H. \& Chitsabean, P. (2017). The influence of neurodevelopmental impairment on youth crime. (In S. Bailey, P. Tarbuck \& P.

Hughes, N., Williams, W., Chitsabesan, P., Walesby, R., \& Mounce, L. (2012). Nobody made the connection: Neurodisability in the youth justice system. Office of the Children's Commissioner for England.

Human Rights Watch. (2018). "I needed help, instead I was punished" abuse and neglect of prisoners with disabilities in Australia. Retrieved July 20, 2019 from Human rights Watch Website https:/www.hrw.org/ report/2018/02/06/i-needed-help-instead-i-was-punished/abuse-and-neglect-prisoners-disabilities

Kippin, N. R., Leitão, S., Watkins, R., Finlay-Jones, A., Condon, C., Marriott, R., Mutch, R., \& Bower, C. (2018). Language diversity, language disorder, and fetal alcohol spectrum disorder among youth sentenced to detention in Western Australia. International Journal of Law and Psychiatry, 61, 40-49. https:// doi.org/10.1016/j.ijlp.2018.09.004.

Lennox, C. (2014). The health needs of young people in prison. British Medical Bulletin, 112, 17-25. https:// doi.org/10.1093/bmb/ldu028.

Lennox, C., O'Malley, K., Bell, V., Shaw, J., \& Dolan, M. (2015). The validity and clinical utility of the Massachusetts Youth Screening Instrument-Version 2 (MAYSI-2) in the UK. Criminal Behaviour and Mental Health, 25, 207-219. https://doi.org/10.1002/cbm.1924.

Lerner, M. D., Haque, O. S., Northrup, E. C., Lawer, L., \& Bursztajn, H. J. (2012). Emerging perspectives on adolescents and young adults with high-functioning autism spectrum disorders, violence, and criminal law. The Journal of the American Academy of Psychiatry and the Law, 40, 177-190.

Lindsay, W., Olley, S., Baillie, N., \& Smith, A. (1999). Treatment of adolescent sex offenders with intellectual disabilities. Mental Retardation, 37, 201-211.

Linhorst, D. M., McCutchen, T. A., \& Bennett, L. (2003). Recidivism among offenders with developmental disabilities participating in a case management program. Research in Developmental Disabilities, 24, 210-230. https://doi.org/10.1016/S0891-4222(03)00029-5.

Longstaffe, S., Chudley, A. E., Harvie, M. K., Markesteyn, T., Neault, D., \& Brown, T. (2018). The Manitoba youth justice program: Empowering and supporting youth with FASD in conflict with the law 1. Biochemistry and Cell Biology, 96, 260-266. https://doi.org/10.1139/bcb-2017-0078.

Martin, G., \& Johnson, C. L. (2005). The boys totem town neurofeedback project: A pilot study of EEG biofeedback with incarcerated juvenile felons. Journal of Neurotherapy, 9, 71-86. https://doi.org/10. 1300/J184v09n03_05.

Maughan, B. (2017). Origins of offending in young people. In S. Bailey, P. Tarbuck, \& P. Chitsabesan (Eds.), Forensic child and adolescent mental health: meeting the needs of young offenders (pp. 11-23). Cambridge University Press. https://doi.org/10.1017/9780511777127.

McCausland, R., \& Baldry, E. (2017). 'I feel like I failed him by ringing the police': Criminalising disability in Australia. Punishment and Society, 19, 290-309. https://doi.org/10.1177/1462474517696126.

McKenzie, K., Paxton, D., Michie, A., Murray, G., Murray, A., \& Curtis, J. (2012). Screening with young offenders with an intellectual disability. Journal of Forensic Psychiatry and Psychology, 23, 676-688. https://doi.org/10.1080/14789949.2012.733723.

McSherry, B., Baldry, E., Arstein-Kerslake, A., Gooding P., McCausland R. \& Arabena K. (2017). Unfitness to plead and indefinite detention of persons with cognitive disabilities. Addressing the legal barriers and creating appropriate alternative supports in the community. Retrieved July 20, 2019, from Melbourne Social Equity Institute, University of Melbourne Web site: https://socialequity.unimelb.edu.au/_data/ assets/pdf_file/0006/2477031/Unfitness-to-Plead-Main-Project-Report.pdf

Moher, D., Liberati, A., Tetzlaff, J., \& Altman, D. G. (2009). Preferred reporting items for systematic reviews and meta-analyses: the PRISMA statement. Annals of Internal Medicine, 151, 264-269. https://doi.org/ 10.1136/bmj.b2535.

Mohr-Jensen, C., Bisgaard, M., Charlotte, B., Kjærsgaard, S., \& Steinhausen, H.-C. (2019). Attention-deficit/ hyperactivity disorder in childhood and adolescence and the risk of crime in young adulthood in a Danish Nationwide Study. Journal of the American Academy of Child and Adolescent Psychiatry, 58, 443-452.

Ögel, K., Karadayi, G., Șenyuva, G., \& Kanoğlu, H. (2012). The reliability and validity study of psychological screening test for adolescents. Düşünen Adam, The Journal of Psychiatry and Neurological Sciences, 25, 8-16. https://doi.org/10.5350/DAJPN2012250101. 
Page, M. J., Higgins, J. P. T., \& Sterne, J. A. C. (2021). Chapter 13: Assessing risk of bias due to missing results in a synthesis. In J. P. T. Higgin, J. Thomas, J. Chandler, M. Cumpston, T. Li, M. J. Page, \& V. A. Welch (Eds.), Cochrane handbook for systematic reviews of interventions version 6.2 (updated February 2021), Cochrane, 2021 Available from www.training.cochrane.org/handbook.

Passmore, H. M., Giglia, R., Watkins, R. E., Mutch, R. C., Marriott, R., Pestell, C., Zubrick, S. R., Rainsford, C., Walker, N., Fitzpatrick, J. P., Freeman, J., Kippin, N., Safe, B., \& Bower, C. (2016). Study protocol for screening and diagnosis of fetal alcohol spectrum disorders (FASD) among young people sentenced to detention in Western Australia. BMJ Open, 6, e012184-e012184. https://doi.org/10.1136/bmjopen-2016012184.

Passmore, H. M., Mutch, R. C., Burns, S., Watkins, R., Carapetis, J., Hall, G., \& Bower, C. (2018). Fetal alcohol spectrum disorder (FASD): Knowledge, attitudes, experiences and practices of the Western Australian youth custodial workforce. International Journal of Law and Psychiatry, 59, 44-52. https:// doi.org/10.1016/j.ijlp.2018.05.008.

Pfeifer, J. E., Winterdyk, J., Hutton, F., Monod de Froideville, S., Banks, C., \& Trounson, J. S. (2018). Indigenous youth crime: An international perspective. In M. Miller \& B. Bornstein (Eds.), Advances in Psychology and Law (pp. 247-284). Springer International Publishing. https://doi.org/10.1007/978-3319-75859-6_8.

Pritchard, D., Graham, N., Penney, H., Owen, G., Peters, S., \& Mace, F. C. (2016). Multi-component behavioural intervention reduces harmful sexual behaviour in a 17-year-old male with autism spectrum disorder: A case study. Journal of Sexual Aggression, 22, 368-378. https://doi.org/10.1080/13552600. 2015.1130269 .

Reid, N. (2018). Fetal alcohol spectrum disorder in Australia: What is the current state of affairs? Drug and Alcohol Review, 37, 827-830. https://doi.org/10.1111/dar.12855.

Reid, N., Kippin, N., Passmore, H., \& Finlay-Jones, A. (2020). Fetal alcohol spectrum disorder: the importance of assessment, diagnosis and support in the Australian justice context. Psychiatry, Psychology and Law, 27, 265-274 DOI 13218719.2020.1719375.

Reid-Cunningham, A. R., \& Fleming, V. C. (2009). Theories of disability: Findings from an analysis of textbooks on human behavior and the social environment. Journal of Human Behavior in the Social Environment, 19, 10-25.

Robinson, L., Spencer, M. D., Thomson, L. D. G., Stanfield, A. C., Owens, D. G. C., Hall, J., \& Johnstone, E. C. (2012). Evaluation of a screening instrument for autism spectrum disorders in prisoners. PLoS One, 7(5), e36078-e36078. https://doi.org/10.1371/journal.pone.0036078.

Seelau, R. (2012). Regaining Control over the children: reversing the legacy of assimilative policies in education, child welfare, and juvenile justice that targeted Native American youth. American Indian Law Review, 37, 63-108.

Snow, P. C., \& Powell, M. B. (2008). Oral language competence, social skills and high-risk boys: What are juvenile offenders trying to tell us? Children and Society, 22, 16-28. https://doi.org/10.1111/j.1099-0860. 2006.00076.x.

Snow, P. C., \& Powell, M. B. (2011). Oral language competence in incarcerated young offenders: Links with offending severity. International Journal of Speech-Language Pathology, 13, 480-489. https://doi.org/ 10.3109/17549507.2011.578661.

Snow, P. C., \& Woodward, M. N. (2017). Intervening to address communication difficulties in incarcerated youth: A phase 1 clinical trial. International Journal of Speech-Language Pathology, 19, 392-406. https://doi.org/10.1080/17549507.2016.1216600.

Swain, N. R., Eadie, P. A., \& Snow, P. C. (2020). Speech and language therapy for adolescents in youth justice: A series of empirical single-case studies. International Journal of Language and Communication Disorders, 55, 458-479. https://doi.org/10.1111/1460-6984.12529.

Tedeschi, F., \& Junewicz, A. (2018). Intellectual disabilities in juvenile justice: the case for screening. Journal of the American Academy of Child and Adolescent Psychiatry, 57, 909-910. https://doi.org/10.1016/j. jaac.2018.07.878.

Thapar, A., Cooper, M., \& Rutter, M. (2017). Neurodevelopmental disorders. The Lancet Psychiatry, 4, 339346. https://doi.org/10.1016/S2215-0366(16)30376-5.

Tinsley Li, S., \& Rogers, S. (2005). Developmental considerations. In M. Hersen (Ed.), Clinician's handbook of child behavioural assessment (pp. 25-61). Elsevier Science \& Technology.

Toombs, M. (2012). Ethical research for Indigenous people by Indigenous researchers. Aboriginal and Islander Health Worker Journal, 36(1), 24-26.

Trevethan, R. (2017). Sensitivity, specificity, and predictive values: Foundations, pliabilities, and pitfalls in research and practice. Frontiers in Public Health, 5, 307. https://doi.org/10.3389/fpubh.2017.00307. 
United Nations. (1989). The United Nations Convention on the Rights of the Child. Retrieved August 5, 2019 from UN Web site https://downloads.unicef.org.uk/wp-content/uploads/2016/08/unicef-conventionrights-child-uncrc.pdf

United Nations. (2006). Convention on the Rights of Persons with Disabilities and Optional Protocol. Retrieved August 5, 2019 from UN Web site https:/www.un.org/disabilities/documents/convention/ convoptprot-e.pdf

United Nations. (2007). United Nations Declaration on the Rights of Indigenous Peoples. Retrieved August 5, 2019 from UN Web site https://www.un.org/development/desa/indigenouspeoples/declaration-on-therights-of-indigenous-peoples.html

Unruh, D. K., Gau, J. M., \& Waintrup, M. G. (2009). An exploration of factors reducing recidivism rates of formerly incarcerated youth with disabilities participating in a re-entry intervention. Journal of Child and Family Studies, 18(3), 284-293. https://doi.org/10.1007/s10826-008-9228-8.

Williams, H. (2012). Repairing shattered lives: Brain injury and its implications for criminal justice. Retrieved August 30, 2019, from Centre for Clinical Neuropsychology Research, University of Exeter. London: Transition to Adult Alliance. Web site https://www.prisonlegalnews.org/media/publications/exeter_univ_ williams brain_injury and criminality_2012.pdf

Yi, S. X., \& Foley, T. (2014). Implementing restorative justice to address indigenous youth recidivism and over-incarceration in the act: Navigating law reform dynamics. Australian Indigenous Law Review, 18, $138-152$.

Young, S., Greer, B., \& Church, R. (2017). Juvenile delinquency, welfare, justice and therapeutic interventions: A global perspective. BJPsych Bulletin, 41, 21-29. https://doi.org/10.1192/pb.bp.115.052274.

Young, S., Gudjonsson, G., Chitsabesan, P., Colley, B., Farrag, E., Forrester, A., Hollingdale, J., Kim, K., Lewis, A., Maginn, S., Mason, P., Ryan, S., Smith, J., Woodhouse, E., \& Asherson, P. (2018). Identification and treatment of offenders with attention-deficit/hyperactivity disorder in the prison population: A practical approach based upon expert consensus. BMC Psychiatry, 18, 281-297. https://doi. org/10.1186/s12888-018-1858-9.

Young, S., Gudjonsson, G., Misch, P., Collins, P., Carter, P., Redfern, J., \& Goodwin, E. (2010). Prevalence of ADHD symptoms among youth in a secure facility: The consistency and accuracy of self- and informant-report ratings. Journal of Forensic Psychiatry and Psychology, 21, 238-246. https://doi.org/ 10.1080/14789940903311566.

Publisher's note Springer Nature remains neutral with regard to jurisdictional claims in published maps and institutional affiliations.

Lorelle Holland is a proud Mandandanji woman. She is an Associate Lecturer at the School of Nursing Midwifery and Social Work and is an Affiliate Associate Lecturer in the Poche Centre for Indigenous Health at the University of Queensland (UQ). She has worked as a Registered Nurse for over three decades in the health care industry in varied clinical, management, education, and research roles. Lorelle completed her Master of Public Health Degree (Field of Indigenous Health) at UQ in 2020. Lorelle is currently enrolled in $\mathrm{PhD}$ studies in the Medicine Faculty at UQ and hopes to explore critical race theory and decolonising research methodologies, to improve holistic assessments to identify complex health needs and to co-design culturally appropriate diversionary pathways to reduce youth detention rates of Aboriginal and Torres Strait Islander youth.

Natasha Reid is a research fellow and clinical psychologist at the University of Queensland, Child Health Research Centre. Her research is focused on prevention, assessment, diagnosis and treatment of fetal alcohol spectrum disorder. Natasha also has clinical experience providing treatment for children, young people and their families with a range of other neurodevelopmental conditions.

Andrew Smirnov is a Senior Lecturer and Director of Research Training at the University of Queensland's School of Public Health. He lectures in substance use from a public health perspective. His research interests include substance use epidemiology over the life course, Indigenous health, the reduction of drug-related harm, treatment and prevention of viral hepatitis, and the health and wellbeing of people who are incarcerated. 


\section{Affiliations}

\section{Lorelle Holland $^{1,2,3}$ • Natasha Reid ${ }^{4}$ - Andrew Smirnov ${ }^{5,6}$}

Natasha Reid

n.reid1@uq.edu.au

Andrew Smirnov

a.smirnov@uq.edu.au

1 School of Public Health, Faculty of Medicine, The University of Queensland, St Lucia, Queensland 4006, Australia

2 School of Nursing, Midwifery and Social Work, Faculty of Health and Behavioural Sciences, The University of Queensland, St Lucia, Queensland 4006, Australia

3 UQ Poche Centre for Indigenous Health, The University of Queensland, St Lucia, Queensland 4006, Australia

4 Clinical Psychology, The University of Queensland, Child Health Research, 62 Graham Street, South Brisbane, Queensland 4101, Australia

5 School of Public Health, Faculty of Medicine, The University of Queensland, Herston, Queensland 4006, Australia

6 Research Strategy and Support (Medicine), Faculty of Medicine, The University of Queensland, Herston, Queensland 4006, Australia 